\title{
Linear instability analysis of low-pressure turbine flows
}

\author{
N. ABDESSEMED, S. J. SHERWIN AND V. THEOFILIS \\ Department of Aeronautics, South Kensington Campus, Imperial College London, \\ London SW7 2AZ, UK \\ School of Aeronautics, Universidad Politécnica de Madrid, Pza. Cardenal Cisneros 3, \\ E28040 Madrid, Spain
}

Three-dimensional linear BiGlobal instability of two-dimensional states over a periodic array of T-106/300 low-pressure turbine (LPT) blades is investigated for Reynolds numbers below 5000. The analyses are based on a high-order spectral $/ h p$ element discretization using a hybrid mesh. Steady basic states are investigated by solution of the partial-derivative eigenvalue problem, while Floquet theory is used to analyse time-periodic flow set-up past the first bifurcation. The leading mode is associated with the wake and long-wavelength perturbations, while a second shortwavelength mode can be associated with the separation bubble at the trailing edge. The leading eigenvalues and Floquet multipliers of the LPT flow have been obtained in a range of spanwise wavenumbers. For the most general configuration all secondary modes were observed to be stable in the Reynolds number regime considered. When a single LPT blade with top to bottom periodicity is considered as a base flow, the imposed periodicity forces the wakes of adjacent blades to be synchronized. This enforced synchronization can produce a linear instability due to long-wavelength disturbances. However, relaxing the periodic restrictions is shown to remove this instability. A pseudo-spectrum analysis shows that the eigenvalues can become unstable due to the non-orthogonal properties of the eigenmodes. Three-dimensional direct numerical simulations confirm all perturbations identified herein. An optimum growth analysis based on singular-value decomposition identifies perturbations with energy growths $O\left(10^{5}\right)$.

\section{Introduction}

Direct numerical simulation (DNS) investigations by Wu \& Durbin (2001), Fasel, Gross \& Postl (2003) and Wissink (2003) modelled the flow in a low-pressure turbine (LPT) passage, which is characterized by Reynolds numbers of the order of $10^{5}$. These investigations are based on different simplifications and modellings of the flow and the blade geometry as well as on different conceptual approaches towards the goal of flow control.

Fasel, Gross \& Postl (2003) employed active control using two- and threedimensional DNS in order to shed light on yet unknown instability mechanisms which may successfully improve flow performance in experiments. For laminar inflow 
conditions, the flow along the adverse pressure gradient portion of the suction surface tends to separate. Wu \& Durbin (2001) observed that periodically passing wakes triggered turbulent spots in the boundary layer along the suction surface that completely prevented the flow from separating downstream. Furthermore, vortices appear along the pressure surface, originating from the vortical structures in the periodically passing wakes. These were stretched due to the accelerating flow along the pressure side. As a result of this stretching the structures aligned with the direction of flow. Zaki \& Durbin $(2005,2006)$ suggested that the turbulent spots on the suction surface may be related to transient growth as the result of linear interaction of non-orthogonal eigenmodes. These spots have also been found by $\mathrm{Wu}$ et al. (1999) who performed DNS modelling a flat blade as well as the incoming wake which revealed the connection to classic Kelvin-Helmholtz/Tollmien-Schlichting (e.g. Kleiser \& Zang 1991) and bypass transition mechanisms. Furthermore it was found that instability in the chosen configuration shared principal characteristics with transient growth phenomena in archetypal flat-plate boundary layer and channel flows (Grek, Kozlov \& Ramazanov 1985; Butler \& Farrell 1992; Trefethen et al. 1993; Schmid \& Henningson 2001), such as the formation of longitudinal vortices which are unstable with respect to three-dimensional small-scale perturbations.

While reproducing longitudinal vortex structures by Wu \& Durbin (2001) a different transition scenario was reported by Wissink (2003). At relatively low Reynolds number, the suction-side boundary layer was found to remain laminar up to and including the location of separation. Separation was very pronounced because of the large angle of attack of the incoming flow. Still, the periodically passing wakes were found to intermittently suppress separation and initially transition occurred through a Kelvin-Helmholtz (KH) instability of the separated shear layer. Further transition to turbulence was observed to take place inside the KH rolls. In a follow-up paper by Wissink, Rodi \& Hodson (2006) it was shown that the KH instability was triggered by the large-scale disturbance corresponding to the movement of the wake as a negative jet, while for further transition to turbulence the presence of small-scale fluctuations inside the periodically passing wakes was necessary.

Generally, counter-rotating vorticity can be associated to algebraically growing instabilities in the previously mentioned canonical flows. Both DNS studies by Wu et al. (1999) and Wu \& Durbin (2001) have identified transient growth to be a physical effect of high significance. Consequently, understanding and modelling transient growth mechanisms in this class of flows appears to be a key to devising successful flow-control methodologies.

Central to all of these investigations are the mechanisms that describe the threedimensional nature of the instabilities of this class of flows. The computational effort that underlies three-dimensional DNS renders this numerical approach accessible only to large-scale facilities and is certainly inappropriate for parametric studies.

However, gaining knowledge about the fundamental eigenspectra that describe the instabilities of LPT flows has been a promising strategy in understanding transition in complex flows.

Initially, an alternative more efficient methodology based on BiGlobal stability analysis for non-parallel flows (Theofilis 2003) has been chosen in the current research effort, following the successful investigations that revealed the eigenspectra of the wellknown (bluff body) flow past a cylinder (Barkley \& Henderson 1996) and NACA-0012 airfoil at an angle of attack (Theofilis, Barkley \& Sherwin 2002), both being related with the LPT-blade. 
Following the methodology of those studies, the present work focuses on a moderate-Reynolds-number range, where the onset of primary and secondary bifurcations is expected.

(In)stability features that can be identified by computing the associated eigenmodes may well be capable of persisting throughout higher-Reynolds_number regimes. Two examples include three-dimensional instability in the two-dimensional (closed) liddriven cavity flow (Theofilis 2000; Albensoeder, Kuhlmann \& Rath 2001; Theofilis, Duck \& Owen 2004), where the frequency of the leading eigenmodes remains practically constant well into the linearly unstable flow regime as well as (open) flow in the wake of a cylinder, where the primary low-Reynolds-number instability associated with the von Karman vortex street leads to flow shedding at a relatively constant frequency (Abdessemed et al. 2008) as the Reynolds number increases.

Two different applications of linear stability analyses in inflectional profiled flows are the low-Reynolds-number investigations by Blackburn, Sherwin \& Barkley (2008b) of the flow in a stenosis and Blackburn, Barkley \& Sherwin (2008a) of the flow past a backward-facing step. They have used transient growth analysis at moderate Reynolds numbers to demonstrate the potential of high temporal growth in causing the flow to transition. In the case of the backward-facing step, the temporal growth is high enough to lead to three-dimensionality already before the asymptotic stability takes place. In stenotic flows the transient instabilities are of convective nature and have the potential to be sustained by incoming noise.

Our approach chosen here allows us to employ the exact representation of the involved complex geometry and to determine the instabilities of non-parallel flows. In the present context, only a two-dimensional DNS need be performed, the results of which are analysed with respect to their stability against the full range of threedimensional (spanwise) wavenumbers at each Reynolds number. For that purpose the Arnoldi method is used, as it is an efficient approach to calculate only the most significant eigenvalues and eigenmodes of the spectrum.

The relationship between transient growth and the non-orthogonal properties of the linear operators describing linear flow instability have been investigated by Trefethen et al. (1993) using the concept of pseudo-spectrum. In addition to investigating the development of disturbances based on two- and three-dimensional eigenmodes, we adapt the pseudo-spectrum analysis to the present context in order to explore the potential for transient growth due to non-orthogonality.

In order to obtain the steady and periodic basic states whose stability characteristics are investigated, the numerical solution of the two-dimensional Navier-Stokes equations has been performed by means of Nektar, a DNS solver based on the spectral/hp element method (Karniadakis \& Sherwin 2006).

Making use of the favourable computational properties of this approach the subsequent instability analysis was also performed using a spectral element methodology, which has been shown in the past to be an appropriate (as well as efficient) means to study the stability of flows in complex configurations (Barkley \& Henderson 1996; Theofilis et al. 2002). A preliminary study (Abdessemed, Sherwin \& Theofilis 2004) was devoted to the investigation of different spatial discretization approaches for both the DNS and the BiGlobal stability analysis. Having shown convergence and the beneficial properties of structured as well as unstructured meshes, we adapt a hybrid mesh in the present effort for all analyses.

The paper is outlined as follows: In $\$ 2$ we introduce the numerical methods including the spectral $/ h p$ element approach to determine the basic states and the BiGlobal stability analysis used to determine the instability of the respective base 
flows. After introducing the numerical model in $\S 3.1$, showing the blade geometry and the distcretized domain, $\$ 3.2$ presents the two-dimensional basic states whose stability we will be analysing in the following sections. In that context, $\S 3.3$ presents results of linear stability analysis of steady states, $\S 3.4$ those of Floquet analysis of periodic states, respectively. Besides discussing the eigenspectra and the modes associated to the most significant eigenvalues identified, in $\$ 3.4 .1$ and 3.4 .3 we compare the results to those obtained when simulating the full nonlinear Navier-Stokes equations in $\$ 3.4 .2$ for some example cases. Our investigation will further lead us to examining the solution of a domain comprising two blades, which we present in \$3.4.4. Finally, in $\$ 3.5$ we discuss a pseudo-spectrum analysis, performed in order to demonstrate the sensitivity of the considered eigenvalue problem due to its non-orthogonal properties and show the potential for transient growth. A subsequent optimum growth analysis in $\$ 3.5 .2$ is presented, identifying physical perturbations with the potential for transition. Conclusions based on the results presented are offered in $\S 4$.

The interested reader may find detailed convergence studies regarding extension of the computational domain as well as $h$ and $p$ refinement in the Appendix.

\section{Theory and numerical methods}

\subsection{Governing equations}

The underlying governing equation for the flow considered are the (non-dimensional) Navier-Stokes and continuity equations for incompressible flow, which can symbolically be written as

$$
\begin{aligned}
\partial_{t} \boldsymbol{u}(x, y, z, t) & =-(\boldsymbol{u} \cdot \nabla) \boldsymbol{u}-\nabla p+\frac{1}{R e} \nabla^{2} \boldsymbol{u}, \\
\nabla \cdot \boldsymbol{u} & =0 .
\end{aligned}
$$

Equation (2.1) can be condensed into its nonlinear and linear contributions:

$$
\partial_{t} \boldsymbol{u}=\boldsymbol{N}(\boldsymbol{u})+\boldsymbol{L}(\boldsymbol{u}) .
$$

The vector $\boldsymbol{u}$ is defined as $\boldsymbol{u}=(u, v, w)^{T}$, where the pressure is eliminated by projection onto a divergence free field. We define the Reynolds number as $R e=U_{\infty} c / v$, with $U_{\infty}$ being the inflow velocity magnitude, $c$ the axial blade chord and $v$ the kinematic viscosity.

\subsection{Direct numerical simulation}

The spectral $/ h p$ element method employed throughout this research is related to the finite-element (FE) approach based on a Galerkin projection. In classical FE schemes, the solution $\boldsymbol{q}$ is approximated by low-order polynomials (typically linear or quadratic), whereas local approximations in the spectral $/ h p$ method are defined by polynomials of high-order $p$. Using respectively less elements of larger characteristic size $h$, this method has been shown to yield accurate and efficient results in the related work of Theofilis et al. (2002), where the relation between accuracy and efficiency is based on exponential growth of the convergence rate with increasing $p$ as opposed to computational costs that grow only algebraically. In particular, we use mixed-weight Jacobi polynomials to represent the solution in two dimensions (Karniadakis \& Sherwin 2006). In the three-dimensional simulations, the solution in the third dimension is approximated by Fourier expansion and an appropriate number of 
Fourier modes. An extensive description of the Spectral $/ h p$ element method can be found in Deville, Fischer \& Mund (2002) and Karniadakis \& Sherwin (2006).

\subsection{Linear stability analysis}

Central to BiGlobal linear flow stability ansatz utilized herein is the concept of decomposition of any flow quantity into a steady or time-periodic laminar basic flow upon which small-amplitude, in principle multi-dimensional, disturbances are permitted to develop. In the context in which the basic state is time-independent and homogeneous in its third dimension this concept can be expressed by decomposing the solution, given in the following by (2.4) for the case of incompressible flow:

$$
\boldsymbol{q}(x, y, z, t)=\overline{\boldsymbol{q}}(x, y)+\varepsilon \hat{\boldsymbol{q}}(x, y) \mathrm{e}^{[\omega t+\mathrm{i} \beta z]}+\text { c.c. },
$$

where we have summarized the velocity vector $\boldsymbol{u}$ and the pressure $p$ to $\boldsymbol{q}=(u, v, w, p)^{T} ; \overline{\boldsymbol{q}}=(\bar{u}, \bar{v}, 0, \bar{p})^{T}$ is a steady solution of the two-dimensional continuity and Navier-Stokes equations; and $\hat{\boldsymbol{q}}=(\hat{u}, \hat{v}, \hat{w}, \hat{p})^{T}$ represents the amplitude of the flow perturbation. Complex conjugation (c.c.) is introduced in the ansatz (2.4) in order to account for the fact that both the total field $q$ and the steady basic flow $\bar{q}$ are real, while both the amplitude and the phase functions of the linear perturbations may in principle be complex.

\subsubsection{Transition from two-dimensional steady to two-dimensional periodic flow (first Hopf bifurcation)}

Initially we will be interested in the two-dimensional instability leading the twodimensional steady basic flow through a Hopf bifurcation to a time-periodic state. In this case the spanwise velocity components in (2.4) all simplify to $w=\bar{w}=\hat{w} \equiv 0$. Accordingly, the spanwise wavenumber $\beta$ is zero as well. For details of the instability analysis of flow past a cylinder in this two-dimensional context the reader is referred to Morzynski \& Thiele (1991).

\subsubsection{Transition from two-dimensional steady to three-dimensional flow}

In order to identify transitional states from a two-dimensional to a threedimensional flow, the disturbance is permitted to assume a harmonic expansion in $z$, satisfying the ansatz (2.4) in which the perturbation is now assumed to comprise all three velocity components and is periodic over a domain of spanwise extent $L_{z}=2 \pi / \beta$, with $\beta$ being a real wavenumber parameter. The spanwise basic flow velocity component $\bar{w}$ is again taken to be zero.

\subsubsection{Eigenvalue problem}

Introducing the ansatz (2.4) into the incompressible Navier-Stokes equations a nonlinear (in terms of both $\bar{q}$ and $\hat{\boldsymbol{q}}$ ) a system of equations is obtained. The basic flow terms associated with $\bar{q}$ and satisfying (2.1) and (2.2) are then subtracted out. The resulting system is linearized about $\bar{q}$, assuming that $\epsilon \ll 1$, to obtain a system of linearized equations which is discretized simultaneously in both the $x$ and $y$ spatial directions. This results in the two-dimensional, partial-derivative matrix-eigenvalue problem for a given set of parameters $\beta$ and $R e$ :

$$
\boldsymbol{A}(\beta, R e) \hat{\boldsymbol{q}}=\omega \hat{\boldsymbol{q}} .
$$

The linear operator satisfies the relation $\boldsymbol{A} \equiv \mathbf{N}_{\bar{q}}+\boldsymbol{L}$. After having introduced decomposition (2.4) into the Navier-Stokes equation (2.1) and (2.2) $\mathbf{N}_{\bar{q}}$ represents the linearized contribution of the perturbed system and $\boldsymbol{L}$ the linear. 

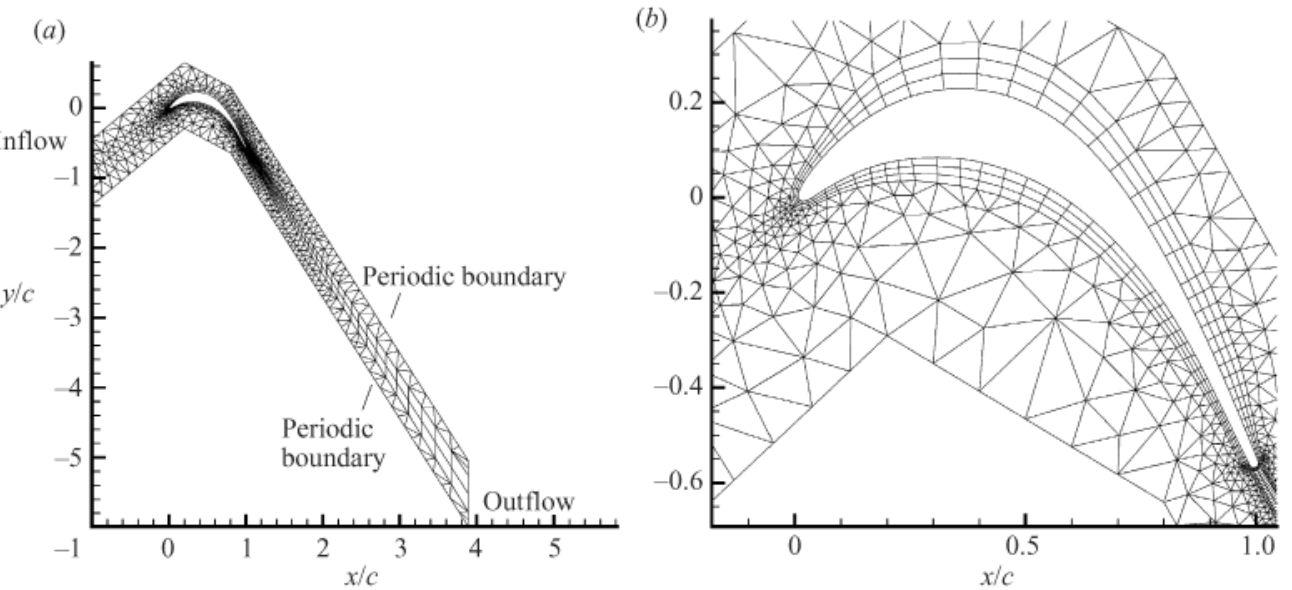

FIGURE 1. (a) Hybrid domain consisting of approximately 2000 elements, indicating boundary conditions. $(b)$ Detailed view of the blade geometry and structured boundary layer.

Furthermore the eigenvalue problem is subject to appropriate boundary conditions over the two-dimensional region $\Omega$, as shown in figure 1 . For the present problem these are the no-slip condition at the surface of the turbine-blade, zero disturbance velocity at the inflow and $\partial \hat{u} / \partial n=\partial \hat{v} / \partial n=0$ at the outflow boundary, as well as periodic connectivity of all flow quantities at the lower and upper boundaries, modelling a cascade configuration as illustrated in figure 1 .

In general the matrix-eigenvalue problem is complex and asymmetric, although here $\bar{w}=0$, which permits a redefinition of $\hat{w}=\mathrm{i} \tilde{w}$, thus resulting in a real eigenvalue problem (Theofilis 2003). This redefinition has the advantage of halving the storage requirements for the solution of the eigenvalue problem which, in turn, is interesting, since the leading dimension of the matrix $\boldsymbol{A}$ is proportional to the degrees of freedom used to discretize the spatial domain. The high-resolution requirements related to sensitivity of our stability results in combination with the relative complexity of the geometry motivate the use of a spatial discretization scheme which provides optimal accuracy at a modest resolution.

Equation (2.5) can be expressed in the time-differential form as

$$
\partial \boldsymbol{q} / \partial t=\boldsymbol{A} \boldsymbol{q} .
$$

In order to solve this equation in an efficient manner the Arnoldi algorithm, which is based on a Krylov subspace iteration method, has been used in combination with the exponential power approach that solves (2.6) explicitly:

$$
\boldsymbol{q}(t+\Delta t)=\boldsymbol{B} \boldsymbol{q}(t)=\boldsymbol{q}(t) \mathrm{e}^{\int_{t}^{t+\Delta t} \boldsymbol{A} d \tau} .
$$

Employing the Arnoldi algorithm on the evolution operator $\boldsymbol{B}(t)$, which evolves $\mathbf{q}(t+\Delta t)$ from $\mathbf{q}(t)$ yields the dominant eigenvalues of $\boldsymbol{B}=\mathrm{e}^{\Delta t \boldsymbol{A}}$, where we have initially assumed $\boldsymbol{A}$ to be independent of time. In order to employ the matrix exponential, the action of the operator can be represented through a time-stepping algorithm in terms of $\boldsymbol{L}$ and $\mathbf{N}_{U}$ (Tuckerman \& Barkley 2000). In that 'matrix-free' framework, neither the operator $\boldsymbol{A}$ nor $\boldsymbol{B}$ has to be constructed, and only their actions are being considered, ultimately enabling us to investigate complex problems in which the large size of $\boldsymbol{A}$ and $\boldsymbol{B}$ would otherwise be constrained by memory limitations. 
The eigenvalues with the largest real part of the matrix $\Delta t \boldsymbol{A}$ are then determined. Since only the stability-significant leading eigenvalues are calculated, the runtime associated with the total process of building the Krylov subspace and obtaining the eigenvalues of the Hessenberg matrix constructed by the iteration is a small fraction of that required by classic methods, such as the QZ algorithm.

\subsection{Floquet stability analysis}

If a Hopf bifurcation exists, the initial steady basic state becomes time-periodic, with a period associated with that of the leading amplified mode identified through (2.4) with $\beta=0$. The resulting unsteady two-dimensional flow may be analysed with respect to its three-dimensional instability by use of Floquet theory. In this context, we are interested in the stability about periodic orbits rather than in the development of perturbed steady-state base flows. The modified algorithm necessary to accomplish this task monitors the $T$-periodic basic state which is then considered to be of the form

$$
\overline{\boldsymbol{q}}=\overline{\boldsymbol{q}}(x, y, t)=\overline{\boldsymbol{q}}(x, y, t+T) .
$$

As the Jacobian matrix is not steady but time-periodic, the stability can not be determined by the eigenvalues of $\boldsymbol{A}$. Rather, stability is determined by the eigenvalues $\mu$ of the monodromy matrix operator $\boldsymbol{B}$ now defined as

$$
\boldsymbol{B}=\mathrm{e}^{\int_{t_{0}}^{t_{0}+T}\left(\mathbf{N}_{\bar{q}}\left(t^{\prime}\right)+L\right) \mathrm{d} t^{\prime} .} .
$$

Equation (2.9) can heuristically be understood as how linearized perturbations evolve around one period $T$ (where the periodic orbit is discretized in, say, $N_{T}$ snapshots). For that reason one has to be aware of the situation in which growth and decay can both occur within one cycle and that by integrating over $\boldsymbol{A}(t)$ only the average growth will be determined. The eigenvalues $\mu$ are called Floquet multipliers; $\mu>1$ describes a growing orbit; $\mu<1$ leads to a limit cycle (see Tuckerman \& Barkley 2000 for further details).

\section{Results and discussion}

\subsection{Numerical model}

LPT flows can be modelled as a cascade of blades, which suggests considering only a single blade and assuming that all flow quantities are periodic in the rotational direction $y$. The periodicity of the flow in both DNS and linear stability analysis is imposed, where this simplification consequently implies that sub-harmonic flow properties are not represented. As we limit ourselves to introducing the discretized domain and the blade geometry in the following, procedures to prove numerical convergence as well as the validity of the model can be found in the Appendix.

Figure 1 illustrates the generated mesh around a T-106/300 LPT blade. As mentioned previously, the numerical method employed permits use of both structured and unstructured meshes, based on triangular and/or quadrilateral elements. The mesh refinement using triangular elements allows a more flexible distribution of mesh density at specific locations of interest such as the trailing and leading edges. In order to yield desired performance of the computations the possibilities of the code were exploited by generating a hybrid mesh consisting of 270 structured elements forming the boundary layer around the blade surface blending into an unstructured mesh for the rest of the field (approximately 2000 elements). The blade geometry itself is approximated by 200 coordinates, which are utilized to perform cubic B-spline 

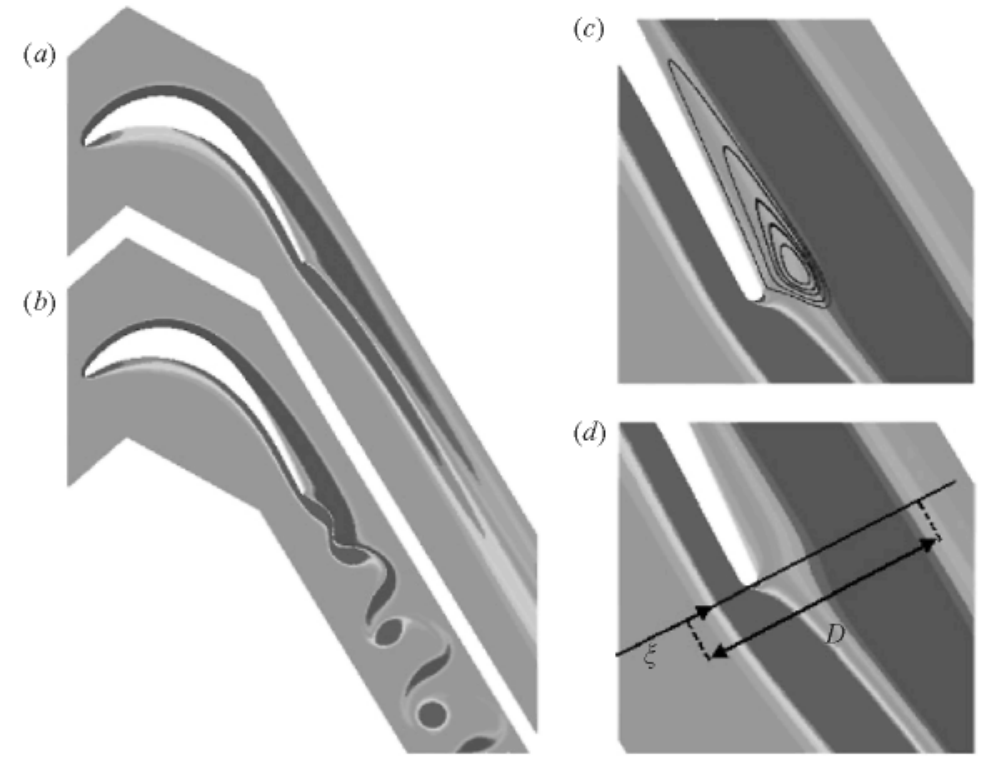

Figure 2. Base flow vorticity for (a) $R e=895$ and (b) $R e=2000$ obtained using 2000 unstructured elements and ninth-order polynomials. (c) Separation bubble and $(d)$ the equivalent bluff body diameter $D$.

interpolations and thus obtain a smooth curved blade surface representation. The degrees of freedom in terms of the polynomial order is $(p+1)^{2}$ for quadrilateral and $(p+1)(p+2) / 2$ for triangular elements, respectively. A comparison with results based on a purely structured mesh can be found in a preliminary study by Abdessemed et al. (2004).

\subsection{Simulation of the two-dimensional basic states}

\subsubsection{Identification of the critical Reynolds number for two-dimensional instability (the first Hopf bifurcation)}

In order to obtain the steady basic states whose linear instability characteristics we are interested in, two-dimensional DNS of the flow have been performed for different Reynolds numbers in the expected range of the primary two-dimensional instability. Figure 2(a) illustrates the vorticity of the computed domain for the flow at $R e=895$, after steady state has been reached. Computations for $R e=905$ lead to qualitatively analogous results, whereas the flow for $R e=906$ becomes time-periodic as illustrated in figure $2(b)$ for a Reynolds number equal to 2000 .

The Hopf bifurcation leading to a transition from a steady to periodic flow at $R e=906 \pm 1$ will be referred to as the first critical Reynolds number denoted by $R e_{c, 2 D}$.

Associating this Reynolds number to an equivalent bluff body diameter $D$ allows the comparison with the well-studied flow past a circular cylinder, whose primary instability takes place at $R e_{c, 2 D}=46$ (Barkley \& Henderson 1996). For this purpose, the equivalent diameter $D$ has been taken as the distance between the local maxima of the velocities on a line as illustrated in figure $2(d)$. This line is located at the very trailing edge and is perpendicular to the flow in the far wake. Denoting the abscissa along the line by a coordinate $\xi$, the equivalent bluff body diameter can be expressed 


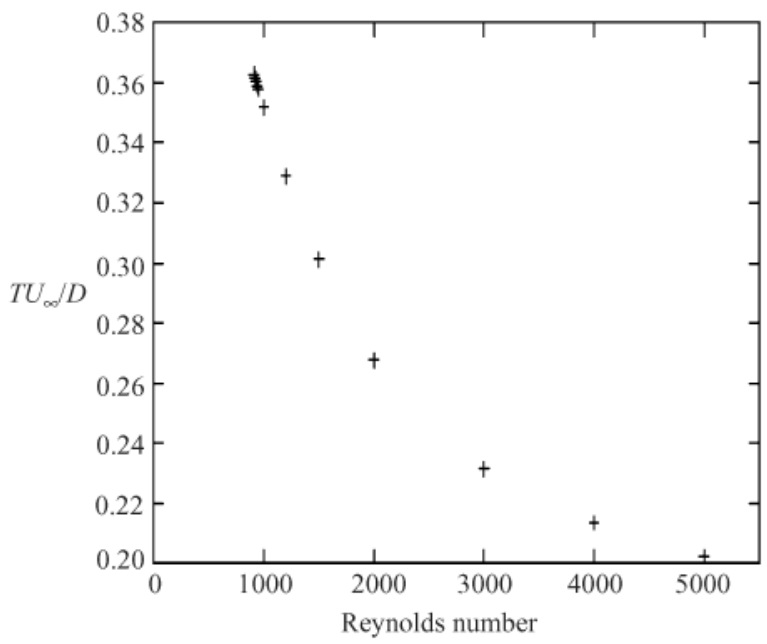

FIGURE 3. Shedding period $T U_{\infty} / D$ over Reynolds number as obtained by two-dimensional DNS.

as

$$
D=\left|\xi\left(\max _{1}|U|\right)-\xi\left(\max _{2}|U|\right)\right|
$$

For base flows close to the transitional state $D \approx 0.3 c$. Identifying the equivalent critical Reynolds number for the flow past a cylinder, where $D \approx 3 c_{c y l}$, leads to a value $R e_{D_{c, 2 D}} \approx 100$, which is about twofold higher than the Hopf bifurcation in the case of the isolated circular cylinder. We assume the asymmetric geometry of our geometry to be responsible for the two-dimensionally stabilizing effects.

As the shedding frequency above $R e_{c, 2 D}$ will play a fundamental role for our Floquet stability investigations, we complete our DNS with figure 3, relating the Reynolds number with the non-dimensionalized shedding period $T$.

\subsection{Linear stability analysis of steady states}

There is no a priori means of asserting whether a two- or three-dimensional perturbation is the relevant ansatz to employ in a flow. Past experience delivers contradictory results in this respect: a two-dimensional (wake) mode is the first to go unstable, turning the basic state unsteady, in both the cylinder (Barkley \& Henderson 1996) and the NACA0012 airfoil (Theofilis et al. 2002). On the other hand, a steady basic state persists up to a Reynolds number $O\left(10^{4}\right)$ in the liddriven cavity (Ghia, Ghia \& Shin 1982; Schreiber \& Keller 1983), while the first three-dimensional instability appears at Reynolds number $O\left(10^{3}\right)$ (Theofilis 2000; Albensoeder et al. 2001). Consequently, in dealing with a new problem, we pursue both paths of investigation, based on two- $(\beta=0)$ and three-dimensional $(\beta \neq 0)$ perturbations of steady flows. Subsequently, the time-periodic flow, which results on account of linear amplification of modes superimposed upon the steady flow, is analysed using the Floquet-theory concept in $\S 3.4$.

\subsubsection{Two-dimensional perturbations}

Initially the analysis is based on (2.4), where we choose $\beta=0$, assuming no perturbation in the spanwise direction. In that context the decomposition represents a purely two-dimensional stability analysis and serves as a test bed against the results obtained via two-dimensional simulations, where we have directly identified $\operatorname{Re}_{c, 2 D}$. 


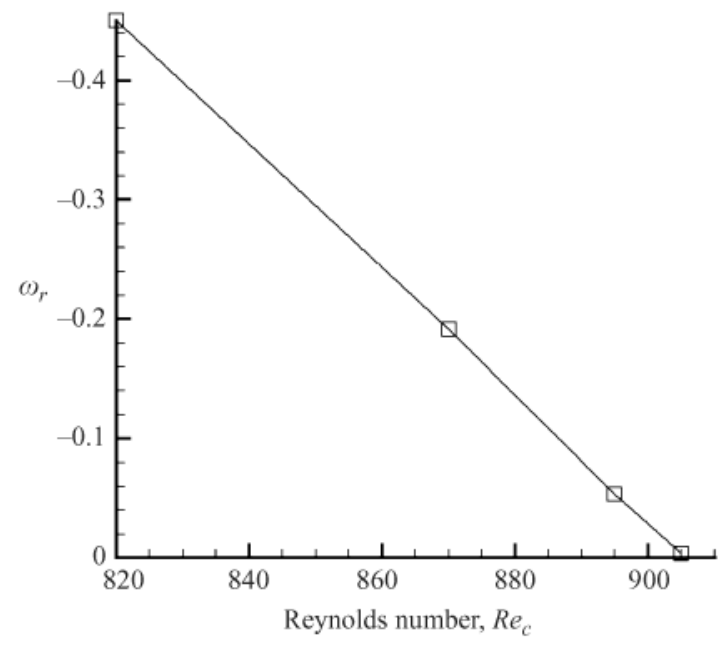

FIGURE 4. Linear correlation between Reynolds number and growth rate (here damping, repectively) of the leading eigenvalue for steady base flows assuming $\beta=0$. With growing Reynolds number, the eigenvalue $\omega_{r}$ tends towards unstable positive values. As the perturbations are purely two-dimensional, the extrapolated value of $R e_{c}=905$ specifies the transition from steady to periodic flow.

Based on that decomposition, the basic states at Reynolds numbers below $\operatorname{Re}_{c, 2 D}$ have been analysed using the Arnoldi algorithm to compute the leading eigenvalues of the two-dimensional system (2.5). Figure 4 summarizes the damping rates for different Reynolds numbers.

As the critical Reynolds number for unsteadiness is approached, increasingly long time integration is required to reach steady state in the base flow computations (since the spectral/ $h p$ method has minimal dissipation). However, a linear extrapolation of $R e$ and the leading eigenvalue, as shown in figure 4, implies that the flow becomes unstable to two-dimensional disturbances at $R e_{c, 2 D} \approx 905 \pm 1$. This result of the BiGlobal stability analysis is consistent with that of the direct simulation.

\subsubsection{Three-dimensional perturbations}

Subsequently, the flow has been analysed with respect to its potential to amplify three-dimensional disturbances, now assuming the decomposition of the flow into a two-dimensional basic state and a three-dimensional perturbation of different spanwise wavelengths $L_{z}=2 \pi / \beta$ based on (2.4). Figure 5 summarizes the results of a parameter studies based on changing $\beta$ at a fixed subcritical Reynolds number $R e<\operatorname{Re}_{c, 2 D}$.

All real parts of the eigenvalues remain negative, converging towards the positive complex plane for increasing Reynolds number and increasing $L_{z}$, demonstrating that a three-dimensional linear instability does not occur below $R e_{c, 2 D}$. The leading eigenvalues obtained for $L_{z}<1$ are real; the ones obtained for $L_{z}>1$ are complex and so are the associated eigenmodes as shown in figure $5(b, c)$.

Interestingly, it appears that the results of the long (spanwise) wavelengths shown in figure 5(c) are associated with the wake pattern, as known from the two-dimensional DNS, and hence we term this mode the wake mode. It is linear amplification of this mode which leads the flow to unsteadiness. Further, the short-wavelength results 

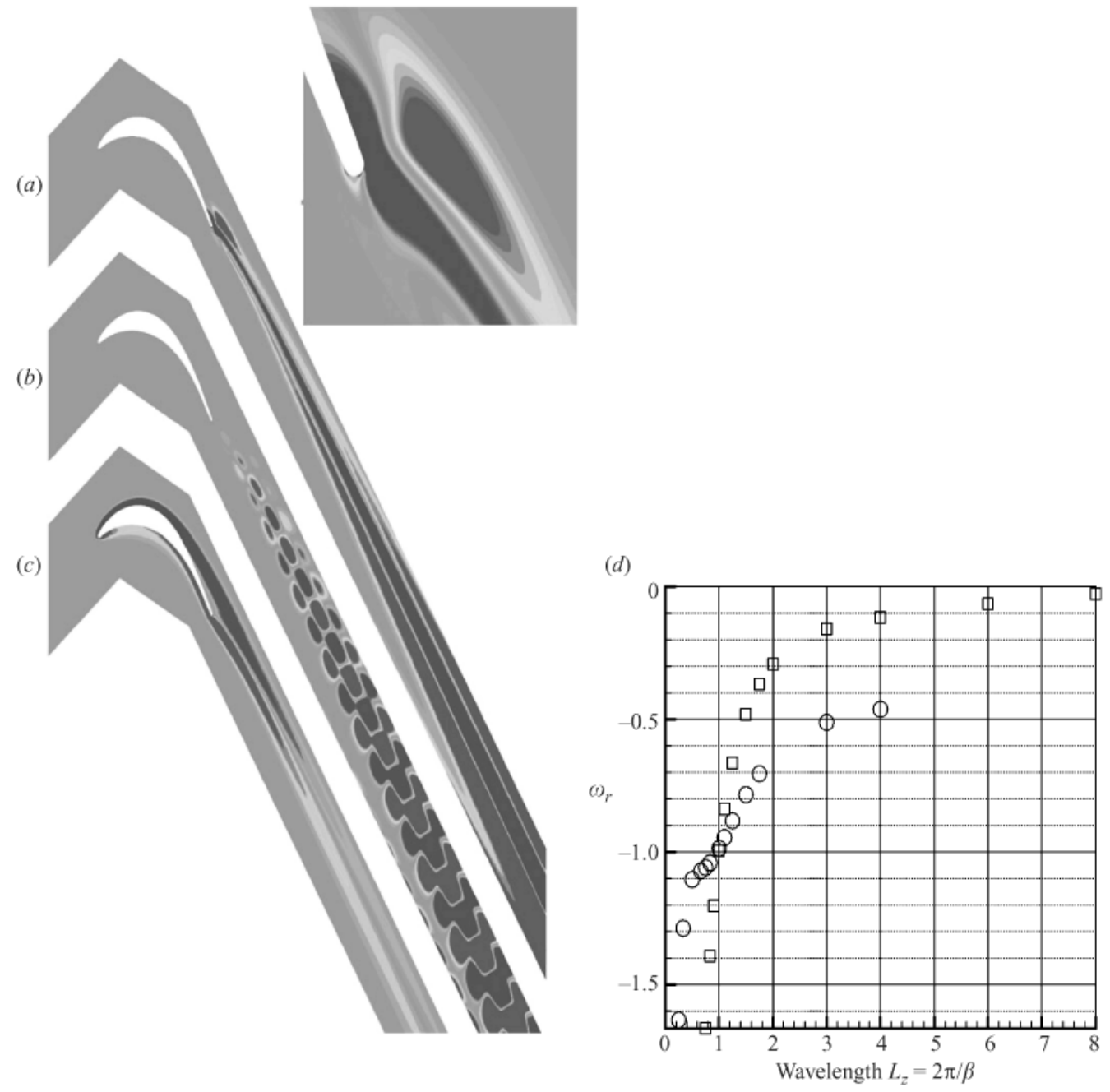

Figure 5. The least stable eigenmodes for $(a) L_{z}=0.25$ (real) highlighting the separation region, (b) $L_{z}=2$ (complex) and their steady base flow (c) at $R e=895$. (d) Damping rates of perturbations at different wavelengths $L_{z}$ in terms of eigenvalues associated to the real (circles) and the complex (squares) eigenmode.

shown in figure $5(b)$ are associated with a mode arising in the trailing-edge region in which separation of the basic state occurs, as seen in figure $2(c)$. We term this the bubble-mode instability.

\subsection{Floquet stability analysis of periodic states}

The previous section demonstrated that the two-dimensional steady flow will become unsteady on account of linear amplification of a two-dimensional mode above $\operatorname{Re}_{c, 2 D}$, while all three-dimensional eigenmodes of the steady state are stable below $\operatorname{Re}_{c, 2 D}$. Once an unsteady two-dimensional flow (of the class shown in figure $2 b$ ) has been set up, Floquet analysis is the tool employed in order to interrogate its three-dimensional instability. 

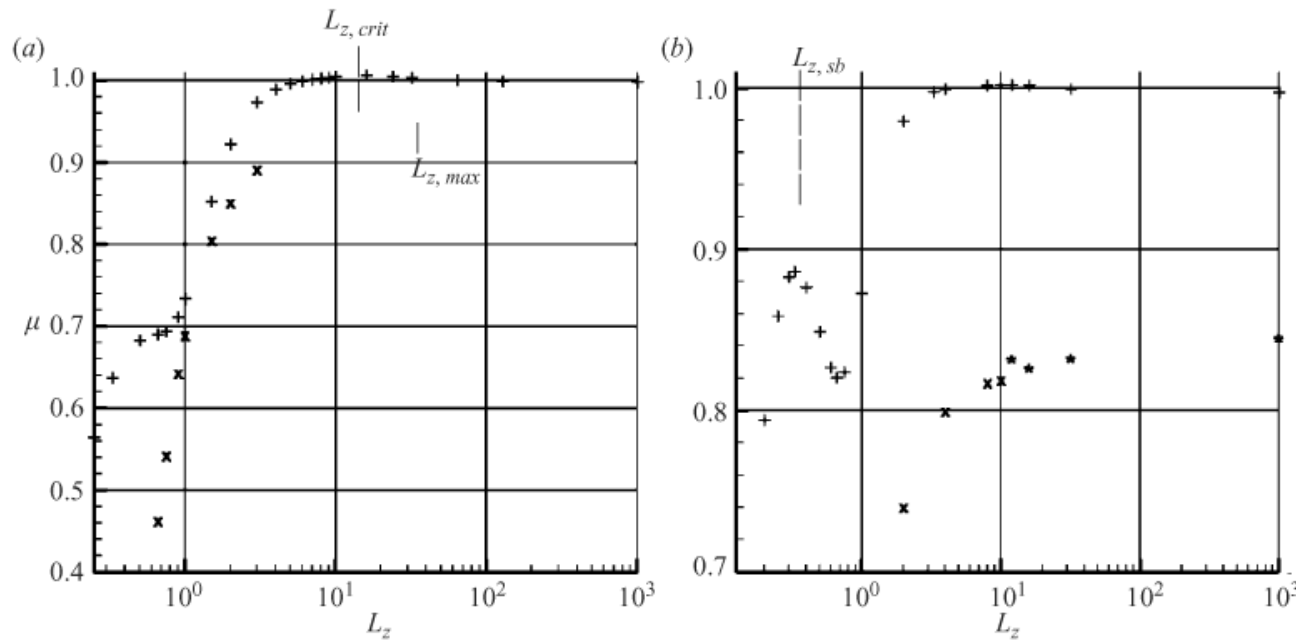

FIGURE 6. Floquet multiplier $\mu$ over wavelength $L_{z}$ for (a) $R e=910$ and $(b) R e=2000$, where + and $*$ are real and $x$ represents complex multipliers; $L_{z, c r i t}$ indicates the onset to three-dimensionality; $L_{z, s b}$ represents the local maximum of the short wavelength perturbation related to the bubble mode.

\subsubsection{Eigenspectra}

In the following, Floquet multipliers $\mu$ have been computed for different spanwise wavelengths $L_{z}$ and different Reynolds numbers at $R e>R e_{c, 2 D}$ at which periodic states are established. Figure 6 summarizes this parameter study with respect to $L_{z}$ for two constant Reynolds numbers right after the two-dimensional transition. We recall that Floquet multipliers below 1 indicate a decaying perturbation, whereas $\mu>1$ are associated with an unstable solution.

By contrast to the steady case, all leading Floquet multipliers, $\mu$, remain real. Since the curve in figure 6 exceeds $\mu=1$ for $L_{z}>5$ and $R e=910$ the flow becomes threedimensionally unstable quite soon after its two-dimensional transition. We denote this critical wavelength at which the flow undergoes its secondary instability $L_{z, \text { crit }}$. This instability is characterized by long-wavelength perturbations as can be seen in the figure for the flow at $R e=910$ and $R e=2000$.

As opposed to other known bluff body flows, the maximum Floquet multiplier $L_{z, \max }$, as highlighted in figure 6 , does not grow significantly with increasing Reynolds number, a finding that will be subject to further discussion. The fact that the instability remains close to the marginal threshold above $\mu=1$ raises the question of whether the numerical model with its highly complex geometry is accurate enough to capture these feeble instability mechanisms both qualitatively and quantitatively. In the Appendix we therefore demonstrate that the computations can be considered accurate enough to determine all instability characteristics as we obtain a solution identifying instabilities whose eigenvalues converge to the third significant figure.

The curves in figure 6 indicating the leading Floquet multipliers for $R e=910$ and $R e=2000$ exhibit a second local maximum evolving from a deflection point with increasing Reynolds numbers. This maximum is associated to short wavelengths and remains stable for all Reynolds numbers investigated.

The identification of $L_{z, \text { crit }}$ for the entire Reynolds number regime under consideration is shown in figure 7. The shaded region in this figure illustrates the unstable regime of the flow for different Reynolds numbers and spanwise 
(a)

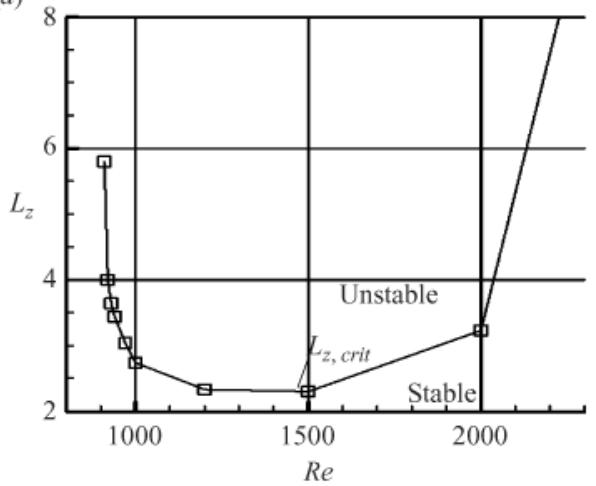

(b)

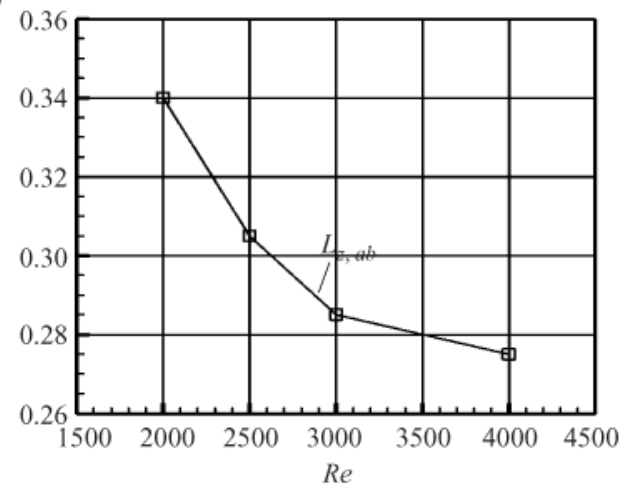

FiguRE 7. (a) The line illustrates the marginal stability at $L_{z, c r i t}$ for different Reynolds numbers; the area above the line represents the unstable regime. $(b)$ Local maximum related to the bubble mode.

perturbations at which the boundary of that region has been obtained by means of interpolation of the above parameter studies for several different Reynolds numbers. For all Reynolds numbers $L_{z, \text { crit }}$ is characterized by long wavelengths above $L_{z, c r i t}=2.3$. As the Reynolds number increases, the critical wavelength $L_{z, c r i t}$ tends to infinity, suggesting that for higher Reynolds numbers the flow remains linearly stable. An approximated relation between $L_{z, s b}$ at the local maximum for different Reynolds numbers complements figure 7.

For $R e=1000$ the energy $\left|(\hat{u}, \hat{v}, \hat{w})^{T}\right|^{2}$ of the Floquet mode, as obtained for these short-wavelength perturbations, is shown in figure 8 alongside the contributions of the velocity components. A concentration of the energy in the region of the separation bubble and the wake can be observed. Despite being stable, the region at the trailing edge supports the possible importance of that mode for further consideration regarding the potential to sustain transient growth due to interaction between stable linear modes. In that context, a transient growth analysis of the associated eigenvalue problem has been performed and will be presented in $\S 3.5$. However, we note that the geometry of the blade considered here is just one of many. Different models with higher inclination angles at the suction surface can have a higher adverse pressure gradient, which possibly destabilizes the bubble and shift the local maximum $L_{z, s b}$ into the unstable regime (Lazaro 2007, private communication), a path that has not been explored in this work.

\subsubsection{Validation using the full DNS solver}

In the following we illustrate the consistency between our results obtained using Floquet stability analysis and three-dimensional DNS based on time marching, where we allow for linear three-dimensional modes to grow. First, the well-known cylinder results by Barkley \& Henderson (1996) have been reproduced; the related numerical experimentation is not shown here. Next we have concentrated on the LPT blade for an example case at $R e=2000$. The DNS results are based on initial conditions at which the obtained two-dimensional Floquet mode has been superposed on the basic state at a low linear amplitude of $10^{-5}$. The solution of the spanwise direction is approximated by only the first non-constant Fourier mode. Since the linearization assumptions of (2.4) are therefore satisfied one expects to obtain the same growth 

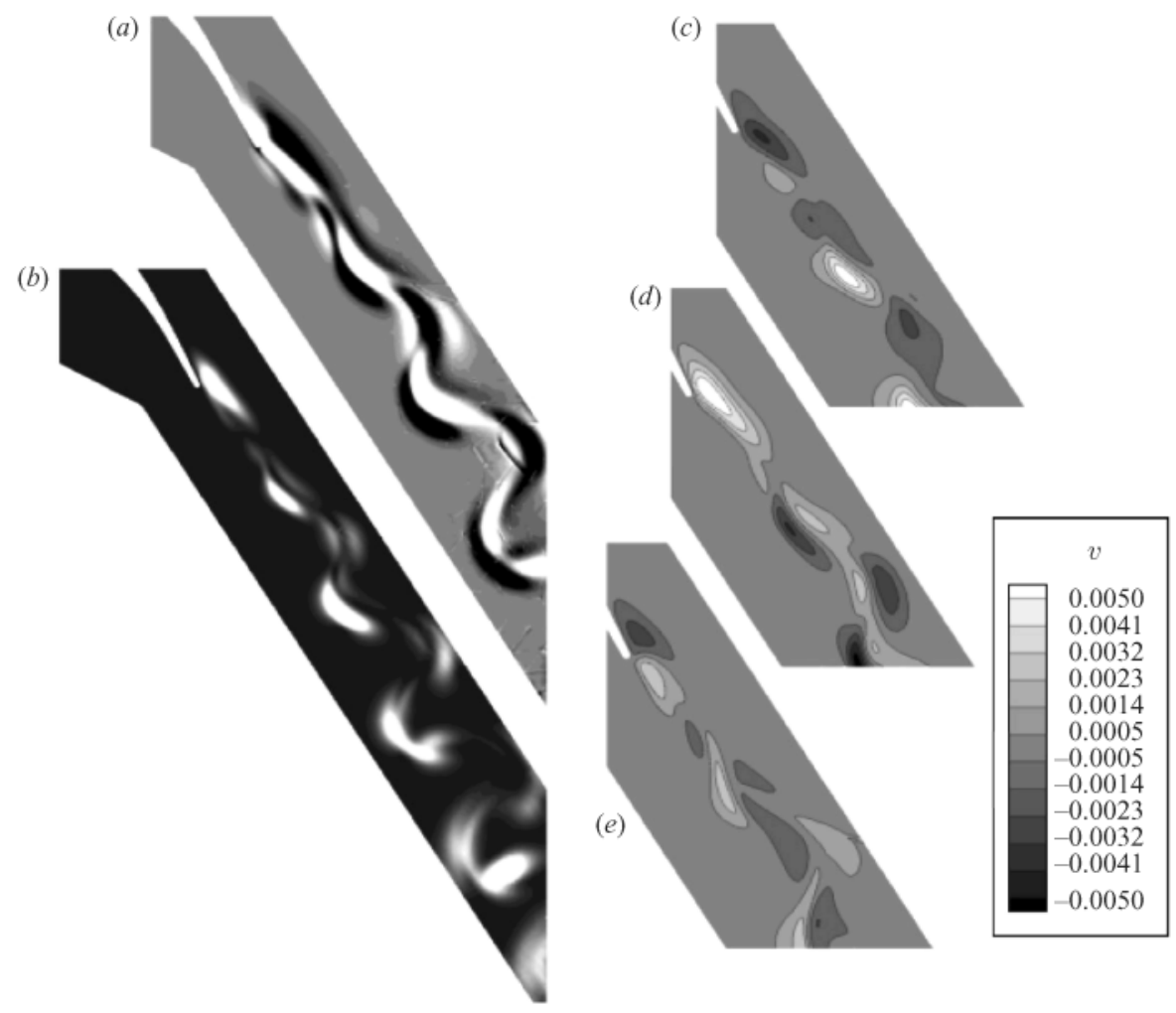

FIGURE 8. Short wavelength mode for $R e=1000$ and $L_{z}=0.33$. (a) Vorticity and (b) concentrated region of the energy at the trailing edge; contributions of the velocity components $(c) \hat{u},(d) \hat{v}$ and $(e) \hat{w}$.

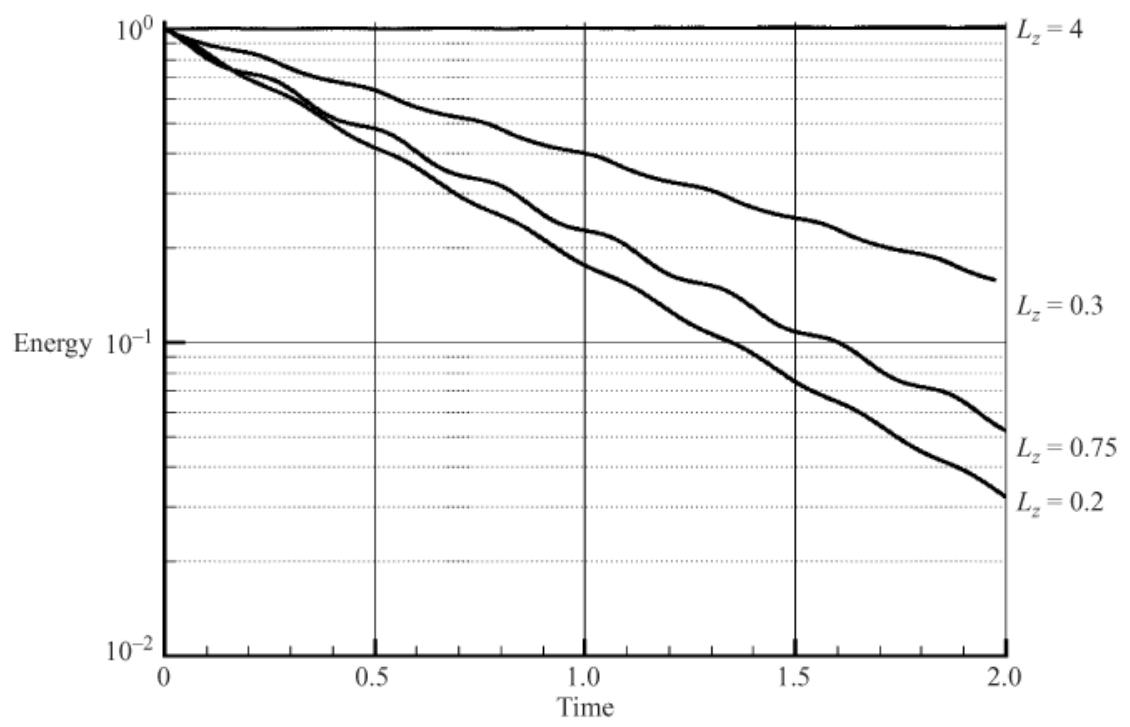

FIGURE 9. Development of the energy of different three-dimensional eigenmodes associated to wavelengths $L_{z}$ obtained by full Navier-Stokes simulation. 


\begin{tabular}{lll}
$L_{z}$ & \multicolumn{1}{c}{$\mu$} & $\mu_{D N S}$ \\
0.2 & 0.795 & 0.797 \\
0.3 & 0.8839 & 0.8846 \\
0.75 & 0.8247 & 0.8261 \\
4 & 1.0004 & 1.001
\end{tabular}

TABLE 1. Comparison of growth rates obtained from Floquet analysis $(\mu)$ and three-dimensional DNS $\left(\mu_{D N S}\right)$.
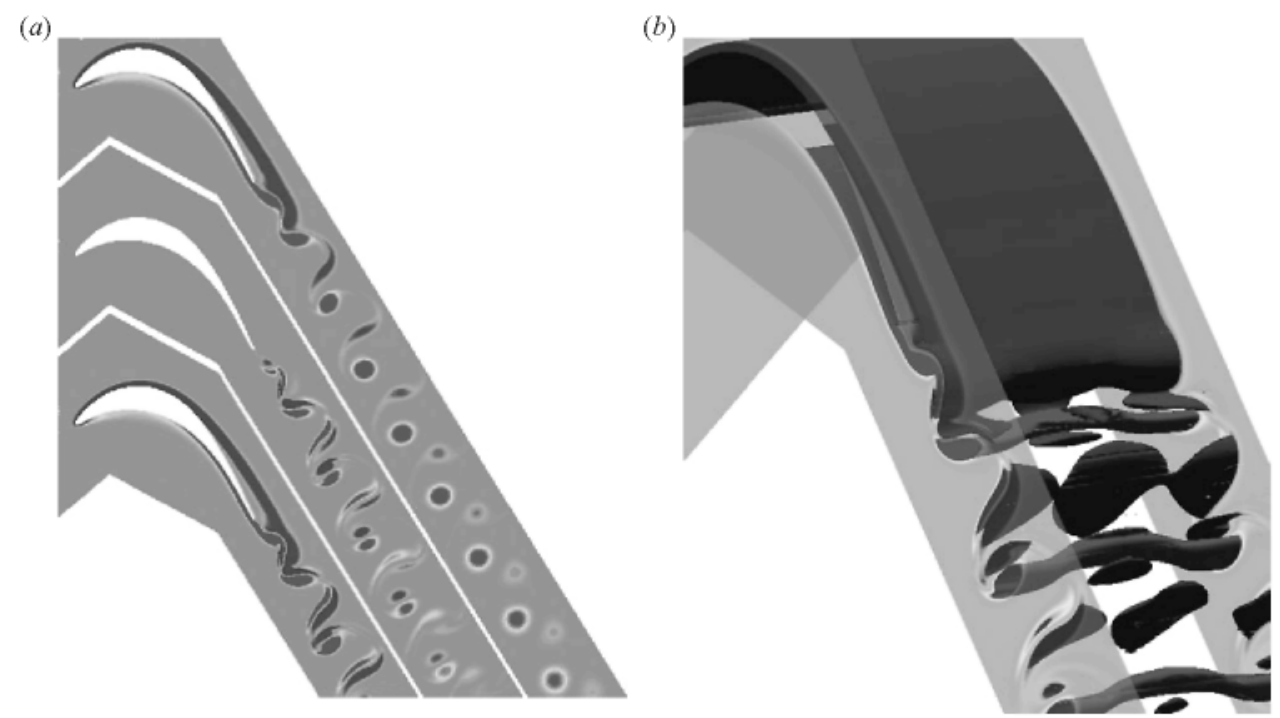

Figure 10. (a) Vorticity fields of base flow (upper), leading long-wavelength eigenmode (middle) and the two superposed fields (lower). (b) Three-dimensional vorticity structure of the superposed field, $R e=2000, L_{z, \max }=12$.

rate as that predicted by Floquet analysis. Figure 9 illustrates the growth of a single and decay of all other modes for different wavelengths $L_{z}$.

The comparison between the damping/growth rate $\mu$ obtained from Floquet analysis and the equivalent measure $\mu_{D N S}$ as obtain by the nonlinear simulation is shown in table 1. For all values under consideration, three-dimensional DNS agrees to the results obtained by Floquet analysis to two significant figures.

\subsubsection{Spatial structure of the unsteady three-dimensional flow field}

The two- and three-dimensional structures of the perturbed basic state can be seen in figure 10. For visualization purposes the combination of base flow and perturbation has been imposed according to $\boldsymbol{q}(x, y, t)+\epsilon \hat{\boldsymbol{q}}(x, y) \mathrm{e}^{[\omega t+\mathrm{i} \beta z]}$, choosing $\epsilon$ to be large enough to illustrate the mode. However, we note that large values of $\epsilon$ do not satisfy the initial assumption of a small perturbations as defined in (2.4). The spatio-temporal symmetry known from mode $\boldsymbol{A}$ and mode $\boldsymbol{B}$ in flow past a cylinder cannot easily be established. A likely reason is the lack of symmetry of the basic flow due to the geometry under investigation. 


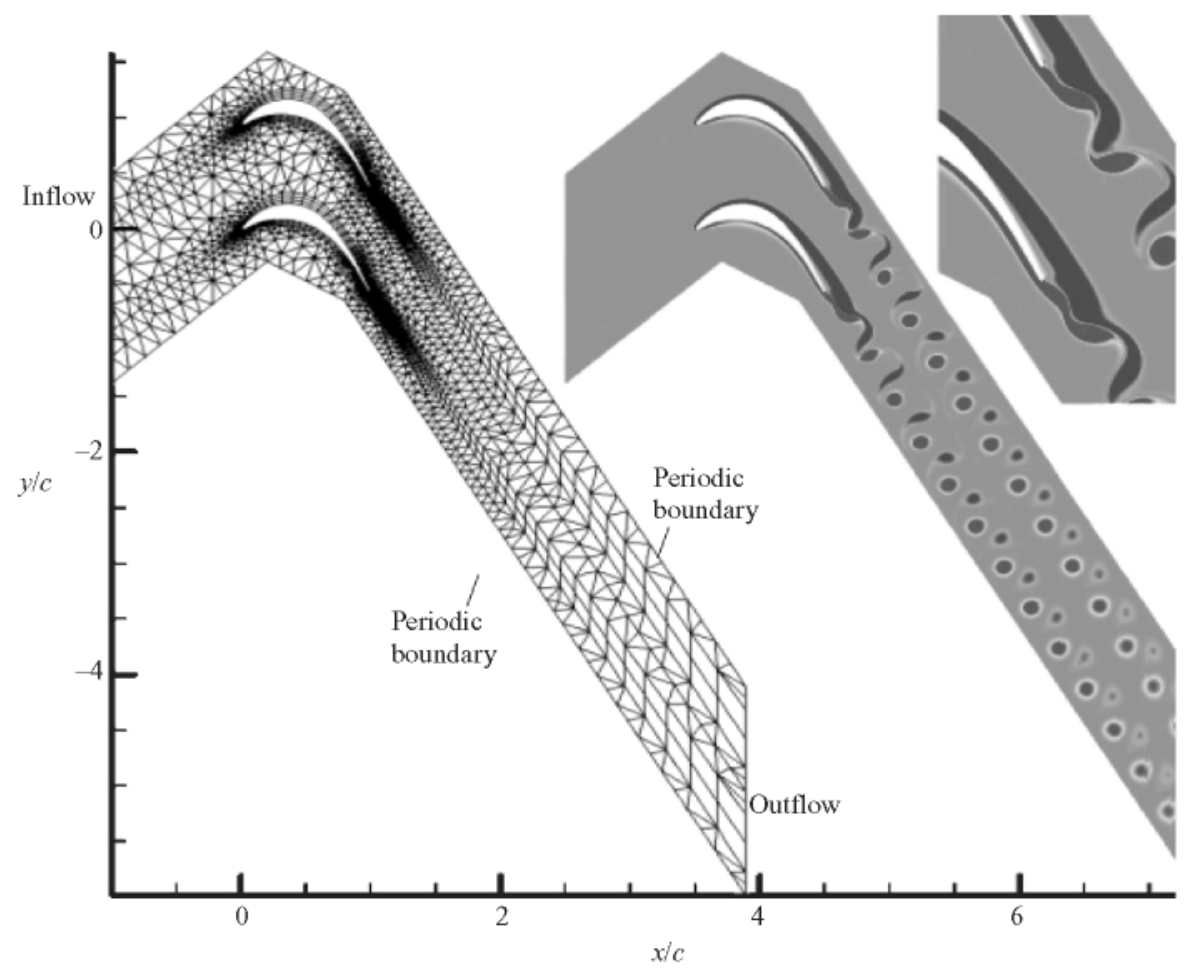

FIgURE 11. Left: extension of the domain comprising two blades with adapted periodic boundary conditions. Right: base flow at $R e=2000$, highlighting the phase lag between the two wakes.

\subsubsection{Investigation of a domain comprising two blades}

As mentioned above, the linear instabilities identified up to now are characterized by very small growth rates for all Reynolds numbers investigated. In order to ensure that these instabilities have been captured accurately, we discuss several numerical convergence scenarios in the Appendix. Furthermore, we now relax the imposed periodic boundary constraints that might possibly influence the instability properties.

A domain consisting of two blades confined to the same periodic boundary conditions has been considered and tested for the set of parameters that played a significant role describing the secondary instability characteristics of the flow. The extended domain is shown in figure 11 together with the computed base state.

There are no major interactions between the two wakes. However, an important observation is a slight phase lag between the shedding of the flow past the upper and the lower blades which shows the potential of rotationally symmetric turbine flows to sustain sub-harmonic effects.

We address the question to what extent those effects may influence the threedimensional instability of the flow in table 2 in which the Floquet multipliers for the two different meshes are shown.

Comparing the original with the extended domain reveals one important qualitative difference: the suppression of sub-harmonic effects yields unstable eigenvalues, while relaxing these constraints leads to a flow that remains linearly stable from a modal perspective. Hence, the previously identified (modal) linear stability depends on the periodic boundary conditions generally assumed in turbine flows. 


$\begin{array}{ccc}L_{z} & \mu & \mu_{\text {ext }} \\ 0.2 & 0.7951 & 0.7939 \\ 0.3 & 0.8839 & 0.8822 \\ 0.4 & 0.8775 & 0.8755 \\ 4 & 1.0021 & 0.9955 \\ 8 & 1.0036 & 0.9965 \\ 16 & 1.0034 & 0.9982 \\ \infty & 0.9997 & 0.9993\end{array}$

TABLE 2. Comparison of Floquet multipliers based on the domain consisting of one blade ( $\mu$, unstable for long-wavelength perturbations) and of two blades $\left(\mu_{e x t}\right)$, stable for all perturbations.

Although we have herewith demonstrated that in the general case the flow is asymptotically stable, it is worth mentioning that under certain working conditions, acoustic effects have been observed to lock the shedding between blades, forcing the flow to be in phase (Lazaro 2007, private communication), so the scenario imposed through the boundary conditions is not of academic value alone. However, we consider the original domain and keep in mind that the following results are based on the model neglecting sub-harmonic effects. Future instability results should therefore have to be compared to cases comprising more than one blade.

The lack of significant linear amplifications within the framework of Floquet stability analysis appears to be an interesting discovery, considering that in bluff body flows asymptotic instability mechanisms describe the onset to three-dimensionality very well.

However, the phenomenon of stable eigenvalues for the entire parameter range of Reynolds numbers and linear perturbations is known from more archetypal flows, such as plane Couette and Hagen-Poiseuille flows (Drazin \& Reid 1981). Furthermore, transient growth mechanisms allow damped disturbances, i.e. stable eigenmodes as identified in the previous sections. For the explanation of the relation between decaying eigenmodes and algebraically growing disturbances the interested reader is referred to Schmid \& Henningson (2001).

As the transient growth mechanisms are related to the non-orthogonal properties of the linear operator $\boldsymbol{A}$, we explore the pseudo-spectrum of the flow in the next section in order to demonstrate the sensitivity of the identified eigenvalues and Floquet multipliers due to the non-orthogonality of the spectrum.

\subsection{Transient growth}

In what follows, we consider transient growth analysis results of a non-parallel flow. While the theory pertinent to one-dimensional ('parallel flow') profiles is well developed (Schmid \& Henningson 2001), little transient growth analysis of essentially non-parallel flows ('BiGlobal transient growth' or 'direct optimal growth analysis) has been reported in the literature; the works of Akervik et al. (2007), Giannetti \& Luchini (2007) and Blackburn et al. $(2008 a, b)$ are first examples in this class of analysis.

\subsubsection{Pseudo-spectrum analysis}

Analysis proceeds by computing the pseudo-spectrum of the matrix discretizing the linearized equations, $\boldsymbol{A}$, via computation of the eigenspectrum $z$ of this matrix $\boldsymbol{A}$, 


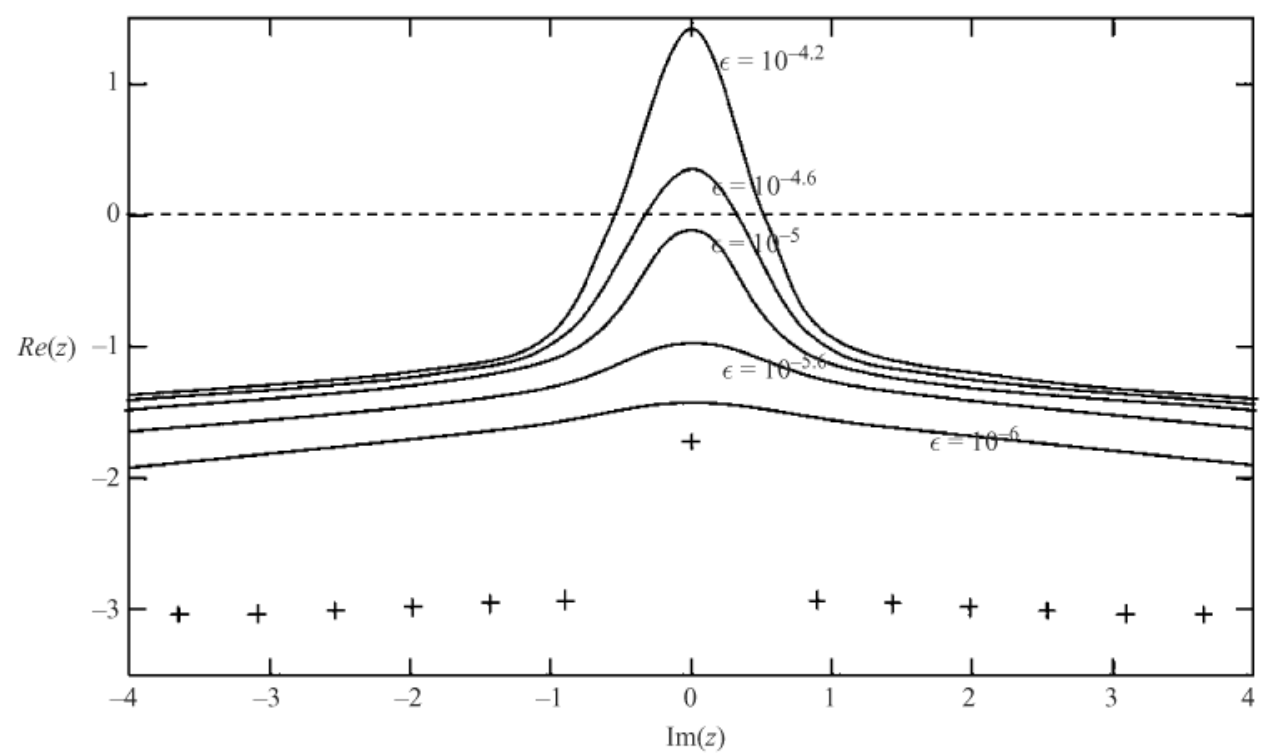

FigurE 12. The BiGlobal pseudo-spectrum of LPT flow at $R e=820, L_{z}=1 / 3 ;+$ represents the eigenvalues of the BiGlobal eigenspectrum of the flow.

perturbed by a small amount, according to

$$
(\boldsymbol{A}+\boldsymbol{E}) \mathbf{x}=z \mathbf{X} .
$$

Here $\boldsymbol{E}$ is of the same dimension as $\boldsymbol{A}$ and contains homogeneously distributed random elements between 0 and $\epsilon$. Schmid \& Henningson (2001) use the definition of the norm $\|\boldsymbol{E}\|$ in order to quantify the perturbation of $\boldsymbol{A}$. The sensitivity of eigenvalues can also be represented using the resolvent $(z \boldsymbol{I}-\boldsymbol{A})^{-1}$, leading to a similar definition of the $\epsilon$-pseudo eigenvalue $z$. In that context a large norm of the resolvent implies strong sensitivity to forcing, which in turn may be related to transient growth.

In the following, results are presented of a linear stability analysis of the perturbed system, using the tools discussed in $\S 2$. Initially, steady flow at a subcritical Reynolds number $R e=820$ is considered, requiring only the solution of the steady linearized eigenvalue problem. Ideally, the full spectrum of the perturbed matrix should be computed within a transient growth analysis, in order to assess the potential of different parts of the eigenspectrum to sustain the transient growth phenomenon. Note that in the case of wall-bounded shear flows, it is the strongly stable members of the eigenspectrum in the intersection of the eigenvalue branches that exhibit the highest sensitivity. In the non-parallel basic flow problem at hand, the computing cost of the Arnoldi approach scales with the dimension of the predetermined Krylov subspace, such that computation of a large number of eigenvalues is impractical. On the other hand, it can be asserted that applied matrix perturbations act on the full spectrum as well, given that we compute the leading (most unstable) eigenvalues. The spectrum of the first few eigenvalues (typically $O(10)$ ) has been obtained at a perturbation of $\epsilon=O\left(10^{-5}\right)$ for an example short spanwise periodicity wavelength $L_{z}=1 / 3$.

All eigenmodes, indicated by + in figure 12, are strongly stable, as discussed in the $\S 3.3$ in which the least damped mode has been identified to correspond to the 'bubble' mode, while the mode next in significance, from a stability analysis point of view, is the 


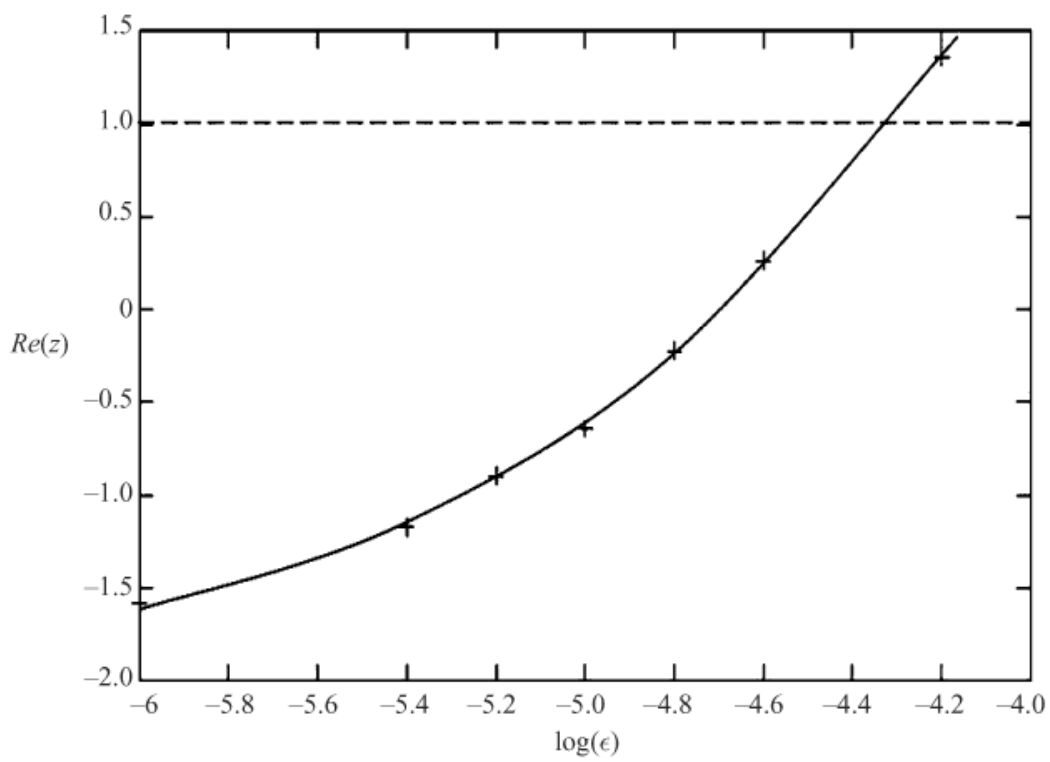

FigURE 13. Dependence of the pseudo-amplification rate on the order of perturbation introduced into the linear matrix operator. Zero-crossing occurs at $\epsilon \approx 10^{-4.7}$.

'wake' mode. The damping rate of the bubble mode is $O\left(10^{-2}\right)$, meaning that if such a perturbation were to be introduced into the flow at a small, linear amplitude, say $A_{0}=O\left(10^{-4}\right)$, it would take a non-dimensional time $\Delta T U / c=O\left(-\ln \left(10^{-12}\right) / 2\right) \approx 14$ (units scaled with the free-stream velocity and the chord length) for the perturbation to subside to machine accuracy levels $A_{1}=O\left(10^{-16}\right)$; if introduced at $A_{0}=O\left(10^{-6}\right)$, the time elapsing for the perturbation to reach machine zero would be $\Delta T U / c=$ $O\left(-\ln \left(10^{-10}\right) / 2\right) \approx 11.5$.

However, a very different picture emerges from the pseudo-spectrum analysis by computing the leading five pseudo-eigenvalues for different perturbations of the order of $\epsilon \in\left[10^{-6}, 10^{-4}\right]$ (units scaled with the free-stream velocity). While the wake disturbances are mildly affected by matrix perturbations in this range, the pseudoeigenvalue corresponding to the bubble mode has a zero crossing. In other words, perturbations $O\left(10^{-4}\right)$ in the original matrix suffice to result in linearly unstable flow through a transient growth mechanism, although linear theory based on the modal eigenvalue problem concept predicts stability. A systematic set of computations of the same phenomenon, scanning the parameter range shown, allows us to plot the boundary curve corresponding to each level of perturbation; which is also shown in figure 12 . While perturbations $O\left(10^{-6}\right)$ result in a more or less uniform shift of the eigenspectrum towards the $\operatorname{Re}(z)=0$ axis, subsequent increases of $\epsilon$ result in a strong destabilization of the bubble mode. Figure 13 shows the monotonic increase of the leading pseudo-eigenvalue with increasing perturbation order. These results may be used to interpolate an approximate matrix perturbation value for which the original linear system becomes unstable, i.e. $\epsilon \approx 10^{-4.7}$.

Finally, initializing a three-dimensional DNS with the most unstable pseudo-mode at an amplitude $O(\ll 1)$ results in the characteristic transient growth curve shown in figure 14; an initial algebraic growth of the perturbation energy is followed by exponential decay. This is the hallmark of transient growth (albeit in a context in which the base flow is two-dimensional), in turn confirming the results presented on 


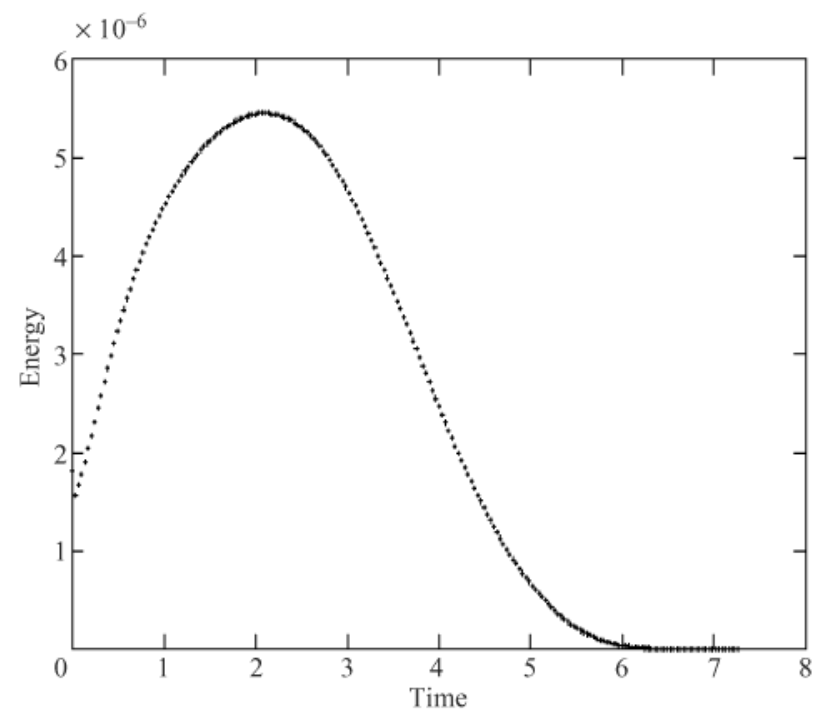

FIGURE 14. Energy growth of the most unstable perturbation at $R e=820, L_{z}=1 / 3, \epsilon=10^{-4}$.

the basis of pseudo-spectrum analysis. The energy growth is relatively small compared to transient growth phenomena in parallel flows, and we confine the investigation to this example case to demonstrate the potential of modes to grow algebraically. Following a newly developed method in recent research efforts (Abdessemed et al. 2008; Blackburn et al. 2008a,b) we employ optimum growth analysis in $\$ 3.5 .2$ in order to identify modes that grow the most in a given time $T$.

\subsubsection{Optimum growth analysis}

The previous pseudo-spectrum analysis was appropriate to demonstrate the potential of random perturbations to exhibit transient behaviour due to interaction of strongly non-orthogonal eigenmodes. As the energy growth based on random perturbations seen in figure 14 is certainly not high enough to lead the flow to transition, we are interested in identifying physical modes that grow the most in a given time $T$; in other words, we seek optimally growing perturbations. For parallel flows these optimum perturbations have been shown to explain transition (e.g. Butler \& Farrell 1992); despite being stable in a classical sense, the disturbance energy might be high enough to trigger further nonlinear mechanisms leading to three-dimensionality. In that respect, flows involving complex geometries have hitherto not been considered due to the limitations of the available numerical methodologies.

In the following, a novel technique based on singular-value decomposition has been applied to determine optimum growing perturbations in complex flow geometries (cf. Sharma et al. 2007; Abdessemed et al. 2008). The methodology has been applied to the flow past a circular cylinder and could successfully identify regions sustaining transient (non-modal) growth exceeding the exponential (modal) development by several orders of magnitude. Similar to seeking leading Floquet multipliers, whose associated modes exhibit maximum exponential growth, we now determine the leading singular values $\sigma$ of $\boldsymbol{B}$ from (2.7), representing the maximum energy gain of an optimum perturbation in a given time $T$, i.e. $\sigma=E(T) / E_{0}$. The singular values are directly related to the eigenvalues of the $\boldsymbol{B}^{*} \boldsymbol{B}$ operator, with $\boldsymbol{B}^{*}$ being the adjoint of $\boldsymbol{B}$. As in the previous section we focus on the steady case below $R e_{c, 2 D}$, referring to our parallel cylinder 


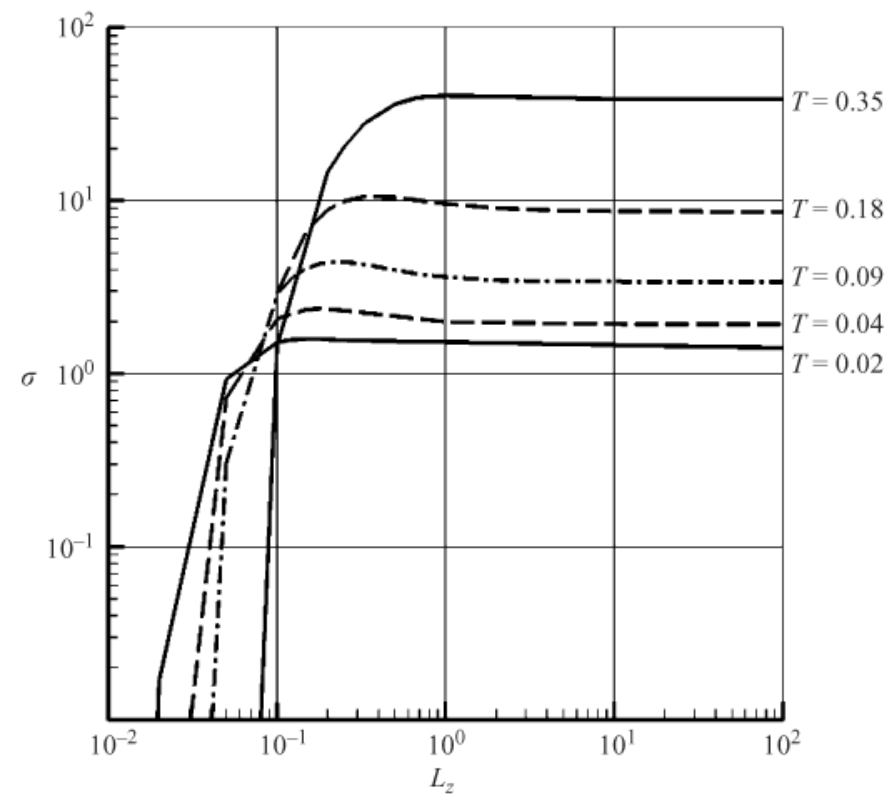

FIGURE 15. Transient growth as function of three-dimensional spanwise wavelengths $L_{z}$ considering different evolution times $T$. As $T$ increases the maximum singular value tends to $L_{z} \rightarrow \infty$.

study in which we established qualitatively similar transient behaviour between the steady and the time-periodic cases in flow past a cylinder. A detailed description of the methodology can be found in the same work.

Figure 15 summarizes the singular values as function of spanwise wavelength $L_{z}$ for different evolution times $T$. The maximum singular value tends to $L_{z} \rightarrow \infty$ with increasing time $T$, indicating that in the long term it is the two-dimensional perturbations that are characterized by the most energy growth, which is consistent with the fact that the flow will undergo a two-dimensional transition at $R e_{c, 2 D}$. We therefore perform a parameter study for that particular two-dimensional case (i.e. $\beta=0$ ), whose result can be seen in figure 16 for different Reynolds numbers. As the Reynolds number approaches the two-dimensional Hopf bifurcation, the leading optimum perturbation grows up to five orders of magnitudes within several nondimensional time units. The associated perturbations are shown in figure $17(a, b)$ for different times $T$. A spatially periodic pattern around the shear layers can be observed, an interesting result considering that this region did not play a significant role in the above eigenmodal investigations. As time passes, the mode develops into a pattern $(c)$ and $(d)$ related to the two-dimensional wake mode and presenting a potential source of disturbances evolving into the two-dimensional instability eigenmode after strong transient growth has occurred. In flow past a cylinder (Abdessemed et al. 2008), the same mechanisms could be observed, showing a relation between the eigenmodal and non-modal perturbations, characterized by transient growth eventually leading the eigenmode to transition in both the two-dimensional steady and the three-dimensional periodic cases. The spatially periodic regions in the shear layer may well be crucial from a control point of view, as they can be associated to frequencies of inflow noise triggering these patterns on the suction surface, causing the periodic flow above $R_{c, 2 D}$ to transition. As opposed to the pseudo-spectrum analysis, where we obtained only 


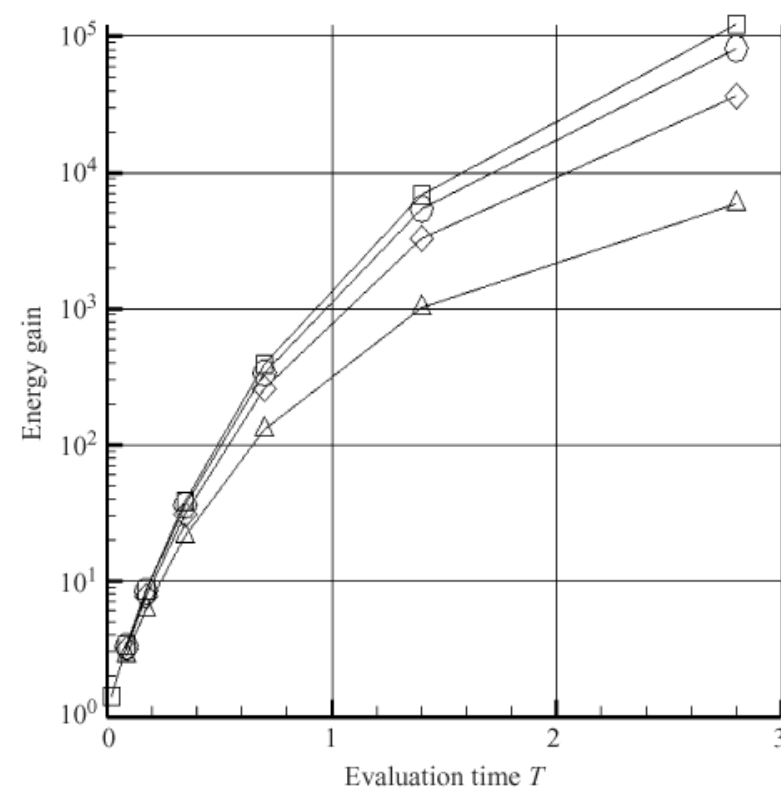

$R e=895 \square$
$R e=870 \bigcirc$
$R e=820 \diamond$
$R e=700 \triangle$

FIGURE 16. As the Reynolds number approaches the two-dimensional Hopf bifurcation, optimum perturbations grow up to five orders of magnitudes within several non-dimensional seconds.

qualitative transient characteristics, the energy gains presented here are large enough to interfere with the base flow even if initial perturbations are small, which shows, for the first time, significant evidence for optimum growth modes leading the LPT flow to transition.

\section{Conclusions}

BiGlobal stability analysis has been employed in order to investigate the eigenspectrum of a T-106/300 LPT blade under uniform incoming flow conditions below and above the transition from steady to unsteady flow. The incompressible twodimensional laminar Navier-Stokes equations have been solved using a spectral $/ h p$ element methodology to obtain steady and periodic base flows at different Reynolds numbers.

Initially, steady basic states were analysed with respect to their two- as well as three-dimensional instability. This study demonstrated that the two-dimensional flow is the least stable and identified the critical Reynolds number for two-dimensional instability to be in the range $R e_{c}=906 \pm 0.5$. In that context, consistency between DNS and linear stability analysis has been demonstrated.

Three-dimensional Floquet analysis has subsequently been utilized to analyse the unsteady basic states above the Hopf bifurcation with respect to three-dimensional instability. These investigations yielded two major results of significance: First, the flow becomes three-dimensionally unstable right after the two-dimensional transition for long-wavelength perturbations of $L_{z, \text { crit }}>5.8 \pm 0.05$ in the range $R e_{c}<R e<910$. For increasing Reynolds numbers this instability is related to $L_{z} \rightarrow \infty$. In other words, the instability is physically relevant only for the moderate-Reynolds-number regime considered in this work. A second region of interest $L_{z, s b}$ which is associated to short wavelength disturbances has been identified. This mode concentrates a significant 

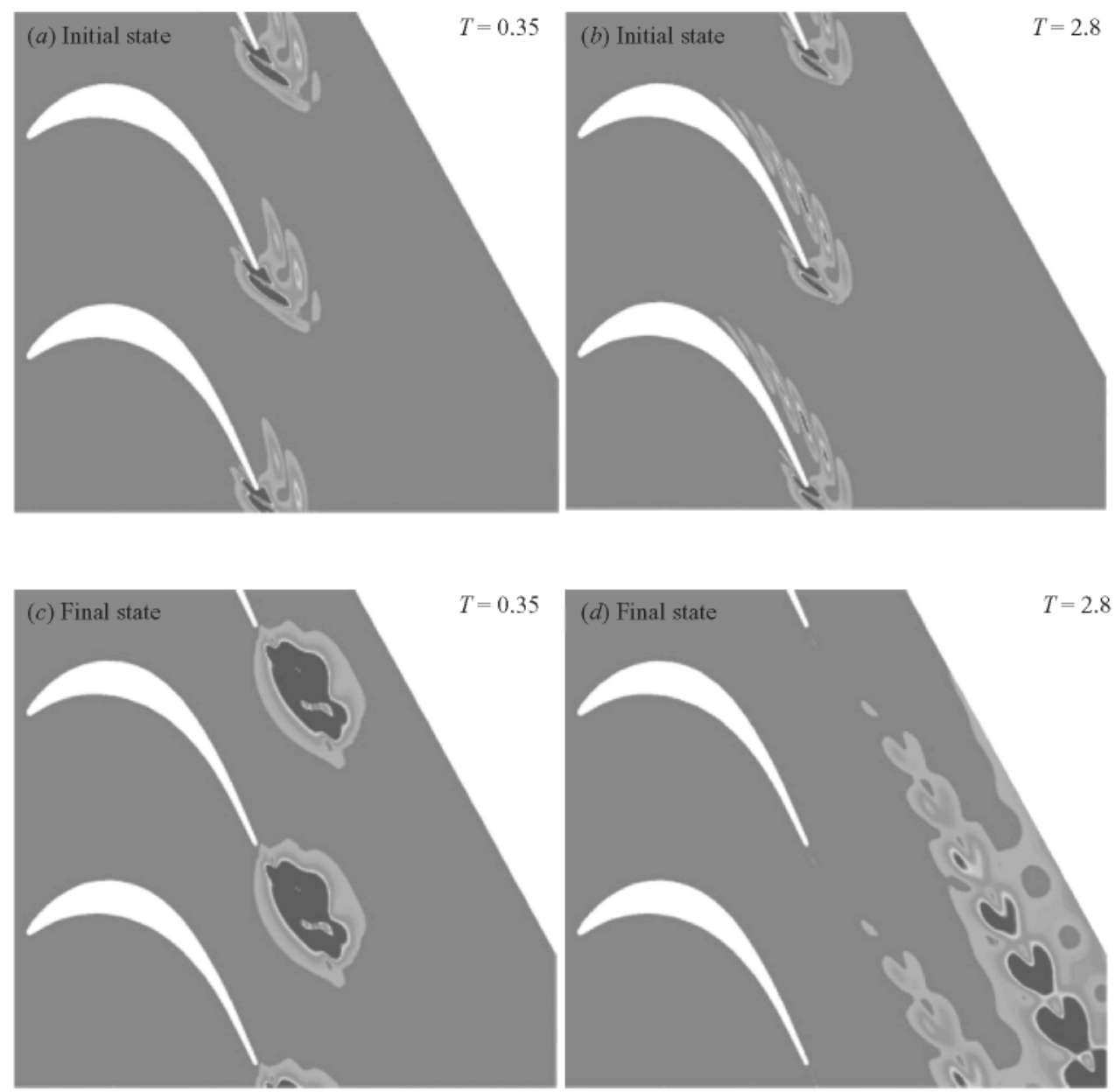

FIGURE 17. Initial and final optimal modes associated to the identified singular values $\sigma$ assuming $\beta=0$ at $R e=895$. In time $T=0.35$, the energy of $(a)$ the left initial mode increases about the singular value of $\sigma \approx 40$ evolving into the pattern of $(c)$ the associated final condition; the right mode's energy evolves in $T=2.8$ from $(b)$ the initial to $(d)$ the final (d) condition while growing about the order $10^{5}$.

In order to investigate the validity of the commonly employed periodic boundary conditions representing the periodic array of turbine blades, we relaxed these constraint and investigated two blades bound to the same conditions. The Floquet analysis showed that the two wakes of the basic states exhibit a phase lag. However, not only does the two-dimensional base flow assume a different solution when the periodic boundary conditions are relaxed, but also, and even more importantly, the previously identified three-dimensional instabilities can be attributed to the supression of sub-harmonic flow features, as all Floquet multipliers could be shown to be stable in the new configuration - for all Reynolds numbers investigated.

Three-dimensional nonlinear simulation initialized with small perturbations based on BiGlobal eigenmodes allowed particular spanwise wavelengths to grow or decay with the respective rates. Again, comparison between DNS and BiGlobal stability analysis delivered coherent results. Summarizing the evidences that BiGlobal stability analysis suggests, it is not exponential growth of linear modes that eventually leads 


$\begin{array}{lccccc}\text { Element count } & \begin{array}{c}\text { Leading } \\ \text { eigenvalue }\end{array} & \begin{array}{c}\text { Damping } \\ \text { rate } \omega_{r}\end{array} & \begin{array}{c}\text { Frequency } \\ \omega_{i}\end{array} & & \\ \text { Unstructured } & \omega_{1} & -0.589960 & & p & \mu_{r} \\ \quad \text { Grid } & \omega_{2} & -1.04967 & \pm 1.70023 & 6 & 1.003445 \\ (1586 \text { elements }) & & & & 7 & 1.003857 \\ & & & & 8 & 1.004331 \\ \text { Unstructured } & \omega_{1} & -0.589963 & & 10 & 1.004111 \\ \quad \text { Grid } & \omega_{2} & -1.05098 & \pm 1.70362 & 11 & 1.004243 \\ \text { (2028 elements) } & & & & & \end{array}$

TABLE 3. Left: variation of mesh density and its element distribution versus eigenvalues for $\operatorname{Re}=700, \beta=0$ and $\mathrm{p}=7$; the analysis is based on (2.4) for steady states. Right: variation of the polynomial order $p$ versus obtained real Floquet multipliers for $R e=2000$ and $L_{z}=8$ using the more resolved mesh. The analysis is based on (2.8) and (2.9).

Therefore a route has been taken investigating the flow's potential for transient growth, following a pseudospectra analysis that successfully explains the connection between non-orthogonality and transient growth in different canonical problems, where we encounter a similar situation, in which linear stability analysis predicts stability for all Reynolds numbers represented by damped eigenvalues.

Returning to Reynolds numbers below $R e_{c}$, where the steady state exists, the employed pseudo-spectrum analyses delivered indications of three-dimensional transient growth due to non-orthogonality which has also been independently confirmed using nonlinear simulations, showing temporary energy growth of the identified pseudo-modes. A subsequent optimum growth analysis could successfully quantify the previously highlighted potential for transient growth and identify regions within the shear layers on the suction surface as significant sources for the transient behaviour. Singular values of up to $10^{5}$ within few non-dimensional time units represent an energy gain of the identified modes well capable to trigger nonlinear mechanisms leading to transition.

\section{Appendix. Convergence tests}

\section{A.0.3. A.1 $h$ refinement}

The integrity of the results is demonstrated by employing grids of different densities to obtain convergence, considering the steady case and two-dimensional case, assuming $\beta=0$ in (2.4). In order to ensure adequate $h$ refinement with the unstructured mesh, the number of elements was increased in the area of the trailing edge. Comparisons of the results using the different meshes with various mesh densities can be seen in table 3 at a single $R e=700$.

The differences in the results on the damping rate of the leading eigenvalues, as generated by the the well-resolved unstructured meshes, are $O\left(10^{-6}\right)$. As the flow comes closer to the onset of two-dimensional instability (see also figure 4), convergence is increasingly challenging to obtain.

\section{A.0.4. A.2 p refinement}

The high-order spectral/hp scheme has been applied using different polynomial orders $p$ in order to investigate the correlation between the accuracy of the solution and the chosen polynomial expansion. Our objective was to employ polynomial 

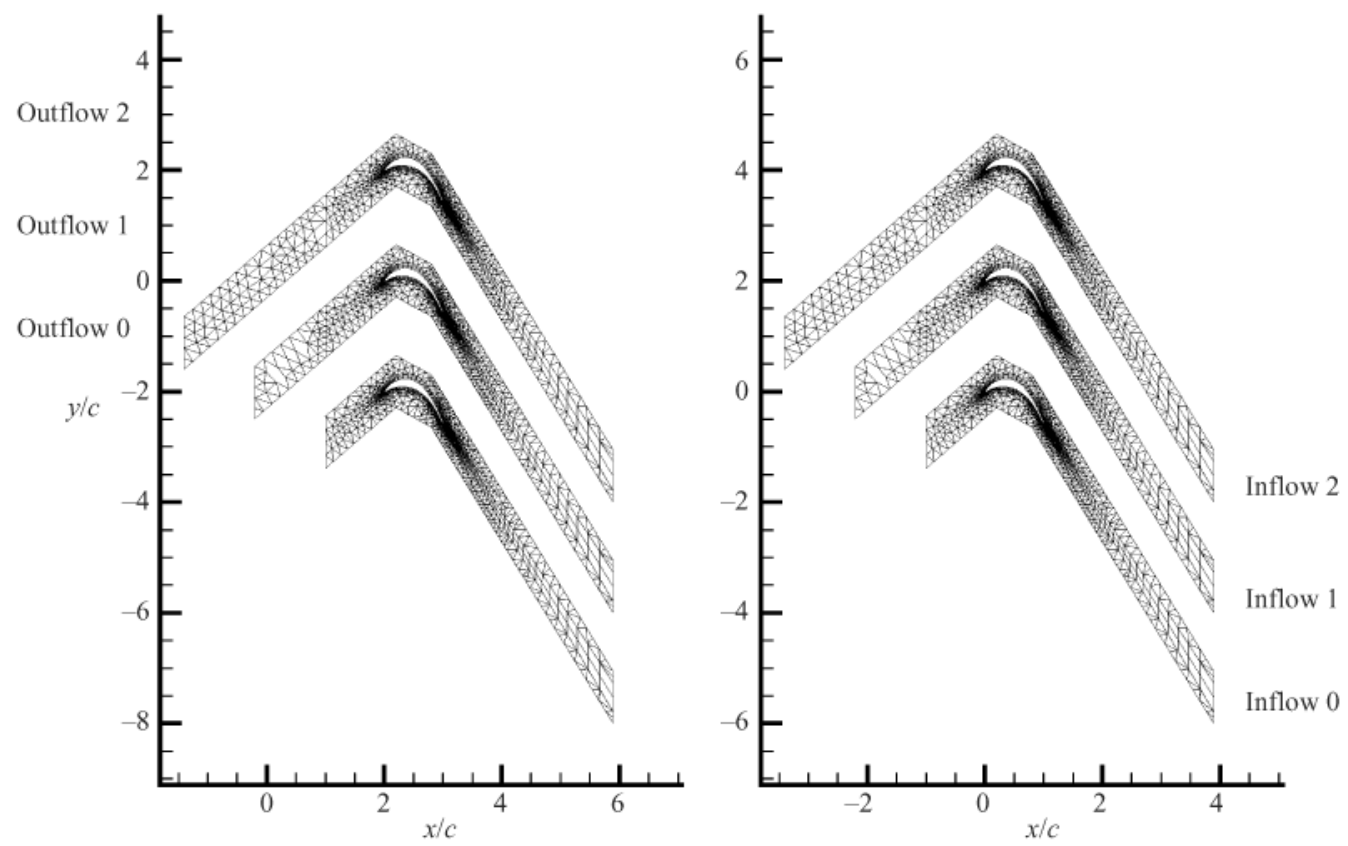

Figure 18. Extension of the computed domain: original mesh (bottom); extended inflow and outflow (top and middle).

orders of sufficient degree to describe the flow physics and yet low enough for the computations to remain efficient. Table 3 also summarizes results of Floquet stability analysis based on (2.8) and (2.9) for different values of $p$ at $R e=2000$, where the flow is time-periodic due to shedding from the trailing edge.

Comparing the Floquet multipliers $\mu_{r}$ with varying polynomial order shows that $p=8$ yields satisfying results to three significant figures in terms of $p$ resolution. All computations have been performed with at least $p=6$, which can be considered as accurate for our purposes.

\section{A.0.5. A.3 Extension of the domain}

While $p$ and $h$ refinements have been used to show numerical convergence, the question of whether the geometrical model of the domain is adequate can be proven by extending the domain appropriately. The distance between the periodic boundaries is predefined, and the the blade's geometry is described by 200 coordinates. The only variable parameter regarding the geometrical model is the distance of inflow and outflow. The extensions of the domain at these boundaries are illustrated in figure 18 .

Table 4 summarizes the results obtained by the Arnoldi algorithm for different extensions of the domain. As it can be seen, the leading eigenvalues do not change significantly with both inflow and outflow extensions. Therefore all following computations have been performed with the baseline domain.

The material is based upon work supported by the Air Force Office of Scientific Research, under grant no. F49620-03-1-0295 to nu-modelling S.L., monitored by Dr T. Beutner (now at DARPA), Lt Col Dr R. Jefferies and Dr J. D. Schmisseur of AFOSR and Dr S. Surampudi of the European Office of Aerospace Research and Development. We would like to thank Dr Richard Rivir and Professor Hugh 


$\begin{array}{lclc}\text { Mesh } & \mu & \text { Mesh } & \mu \\ \text { Outflow 0 } & 1.00135 & \text { Inflow 0 } & 1.00135 \\ \text { Outflow 1 } & 1.00187 & \text { Inflow 1 } & 1.00100 \\ \text { Outflow 2 } & 1.00163 & \text { Inflow 2 } & 1.00142\end{array}$

TABLE 4. Inflow and outflow extensions of the unstructured mesh and the obtained leading Floquet multipliers at $R e=2000$ and $L_{z}=8$.

Blackburn for discussions and many useful comments, as well as the anonymous reviewers whose comments contributed to improving the introduction of our manuscript.

\section{REFERENCES}

Abdessemed, N., Sharma, A., Sherwin, S. \& Theofilis, V. 2009 Transient growth analysis of the flow past a cylinder. Phys. Fluids. 21, 044103.

Abdessemed, N., Sherwin, S. \& Theofilis, V. 2004 On unstable 2D basic states in low pressure turbine flows at moderate Reynolds numbers. Paper 2004-2541. AIAA.

Akervik, E., Hoepffner, J., Ehrenstein, U. \& Henningson, D. S. 2007 Optimal growth, model reduction and control in a separated boundary-layer flow using global eigenmodes. J. Fluid Mech. 579, 305-314.

Albensoeder, S., Kuhlmann, H. C. \& Rath, H. J. 2001 Multiplicity of steady two-dimensional flows in two-sided lid-driven cavities. Theor. Comput. Fluid. Dyn. 14, 223-241.

Barkley, D. \& Henderson, R. 1996 Three-dimensional Floquet stability analysis of the wake of a circular cylinder. J. Fluid Mech. 322, 215 - 241.

Blackburn, H. M., Barkley, D. \& Sherwin, S. J. 2008 a Convective instability and transient growth in flow over a backward facing step. J. Fluid Mech. 603, 271-304.

Blackburn, H. M., Sherwin, S. J. \& Barkley, D. $2008 b$ Convective instability and transient growth in steady and pulsatile stenotic flows. J. Fluid Mech. 607, 267-277.

Butler, K. M. \& Farrell, B. F. 1992 Three-dimensional optimal perturbations in viscous shear flow. Physics of Fluids A 4 (8), 1637-1650.

Deville, M. O., Fischer, P. F. \& Mund, E. H. 2002 High-Order Methods for Incompressible Fluid Flow. Cambridge University Press.

Drazin, P. \& ReID, W. 1981 Hydrodynamic Stability. Cambridge University Press.

Fasel, H., Gross, A. \& Posth, D. 2003 Control of separation for low pressure turbine blades: numerical simulations. In Proceedings of the Global Flow Instability and Control Symposium II (unpublished).

GHia, U., GHIA, K. \& SHIN, C. 1982 High-resolutions for incompressible flow using the NavierStokes equations and a multigrid method. J. Comput. Physics 48, 387-411.

Giannetti, F. \& Luchini, P. 2007 Structural receptivity of the first instability of the cylinder wake. J. Fluid Mech. 581, 167-197.

Grek, H. R., Kozlov, V. V. \& Ramazanov, M. P. 1985 Three types of disturbances from the point source in the boundary layer. In Proceedings of the LaminarTurbulent Transition Symposium $I I$, (ed. V. Kozlov), pp. 267-272.

Karniadakis, G. E. \& Sherwin, S. J. 2006 Spectral/hp Element Methods for CFD, 2nd ed. Oxford University Press.

KLEISER, L. \& ZANG, T. A. 1991 Numerical simulation of transition in wall-bounded shear flows. Annu. Rev. Fluid Mech. 23, 495-537.

Morzynski, M. \& Thiele, F. 1991 Numerical stability analysis of flow about a cylinder. Z. Angew. Math. Mech. 71, T424-T428.

Schmid, P. \& Henningson, D. 2001 Stability and Transition in Shear Flows. Springer.

SChreiber, R. \& Keller, H. 1983 Driven cavity flows by efficient numerical techniques. J. Comput. Phys. 49, 310-333. 
Sharma, A., Abdessemed, N., Sherwin, S. \& Theofilis, V. 2008 Optimal growth modes in flows in complex geometries. In Proceedings of the IUTAM Symposium on Fluid Control and MEMS. (ed. J. F. Morrison, D. M. Birch \& P. Lavoie), vol. 7, pp. 339-343, Springer.

Theofilis, V. 2000 Globally-unstable flows in open cavities. Paper 2000-1965. AIAA.

THEOfilis, V. 2003 Advances in global linear instability of nonparallel and three-dimensional flows. Prog. Aero. Sci. 39 (4), 249-315.

Theofilis, V., Barkley, D. \& Sherwin, S. 2002 Spectral/hp element technology for global flow instability and control. Aero. J. 106, 619-625.

Theofilis, V., Duck, P. W. \& Owen, J. 2004 Viscous linear stability analysis of rectangular duct and cavity flows. J. Fluid Mech. 505, 249-286.

Trefethen, L. N., Trefethen, A. E., Reddy, S. C. \& Driscoll, T. 1993 Hydrodynamic stability without eigenvalues. Science 261, 578-584.

Tuckerman, L. \& Barkley, D. 2000 Bifurcation analysis for timesteppers. In Numerical Methods for Bifurcation Problems and Large-Scale Dynamical Systems (ed. E. Doedel \& L. Tuckerman), vol. 119 , pp. 543-466. Springer.

WIssink, J. G. 2003 DNS of separating, low Reynolds number flow in a turbine cascade with incoming wakes. Intl J. Heat Fluid Flow 24, 626-635.

Wissink, J. G., Rodi, W. \& Hodson, H. P. 2006 The influence of disturbances carried out by periodically incoming wakes in the separating flow around a turbine blade. Intl J Heat Fluid Flow 27, 721-729.

Wu, X. \& Durbin, P. A. 2001 Evidence of longitudinal vortices evolved from distorted wakes in a turbine passage. J. Fluid Mech. 446, 199-228.

Wu, X., JAcobs, R., Hunt, J. \& Durbin, P. A. 1999 Simulation of boundary layer transition induced by periodically passing wakes. J. Fluid Mech. 398, 109-153.

Zaki, T. A. \& Durbin, P. A. 2005 Mode interaction and the bypass route to transition. J. Fluid Mech. 531, 85-111.

ZAKI, T. A. \& Durbin, P. A. 2006 Continuous mode transition and the effects of pressure gradient. J. Fluid Mech. 563, 357-388. 